\title{
A novel adaptive control design method for stochastic nonlinear systems using neural network
}

\author{
Mohammad Mahdi Aghajary ${ }^{1} \cdot$ Arash Gharehbaghi $^{2}$ (D)
}

Received: 27 January 2020 / Accepted: 5 January 2021 / Published online: 18 February 2021

(C) The Author(s) 2021

\begin{abstract}
This paper presents a novel method for designing an adaptive control system using radial basis function neural network. The method is capable of dealing with nonlinear stochastic systems in strict-feedback form with any unknown dynamics. The proposed neural network allows the method not only to approximate any unknown dynamic of stochastic nonlinear systems, but also to compensate actuator nonlinearity. By employing dynamic surface control method, a common problem that intrinsically exists in the back-stepping design, called "explosion of complexity", is resolved. The proposed method is applied to the control systems comprising various types of the actuator nonlinearities such as Prandtl-Ishlinskii (PI) hysteresis, and dead-zone nonlinearity. The performance of the proposed method is compared to two different baseline methods: a direct form of backstepping method, and an adaptation of the proposed method, named APIC-DSC, in which the neural network is not contributed in compensating the actuator nonlinearity. It is observed that the proposed method improves the failure-free tracking performance in terms of the Integrated Mean Square Error (IMSE) by $25 \% / 11 \%$ as compared to the backstepping/APIC-DSC method. This depression in IMSE is further improved by $76 \% / 38 \%$ and $32 \% /$ $49 \%$, when it comes with the actuator nonlinearity of PI hysteresis and dead-zone, respectively. The proposed method also demands shorter adaptation period compared with the baseline methods.
\end{abstract}

Keywords Actuator nonlinearity · Dead-zone - Adaptive neural network dynamic surface control (ANNDSC) Nonlinear stochastic systems · Prandtl-Ishlinskii hysteresis model $\cdot$ Strict-feedback systems

\section{Introduction}

Fault tolerant control systems with actuator failure compensation have received many interests from the researchers of industrial control field over decades [1-7]. Serious studies in computer science have been dedicated to address important theoretical and practical questions, raised in adaptive nonlinear control systems, where dynamic surface control (DSC) method served as a novel useful tool for designing adaptive control systems,

Arash Gharehbaghi

arash.gharehbaghi@liu.se

Mohammad Mahdi Aghajary

maghajary2004@gmail.com

National Iranian Gas Company, Tehran, Iran

2 Department of Biomedical Engineering, Linköping University, Linköping, Sweden especially for nonlinear strict-feedback [8], [9], and fractional-order [10] systems.

An important research question, which was not addressed in those studies [11-21], is effect of stochastic behaviors and Prandtl-Ishlinskii (PI) hysteresis on the system performance. PI or backlash-like hysteresis and dead-zone phenomena are considered as the two important general nonlinearities, seen in the literature. However, a general adaptive control method with the capability of incorporating both stochastic and nonlinear behaviors of the control system, including the joint Prandtl-Ishlinskii hysteresis and dead-zone phenomena, cannot be seen in those studies in an objective way. One of the problems in developing such a generalized method corresponds to stability of the methods at the presence of an unknown nonlinearity.

Dynamic surface method has been employed by several neural network-based methods for nonlinear control systems [9, 12, 21-24]. However, this is not true for stochastic 
nonlinear systems, when general nonlinearities such as PI hysteresis and dead zone appear in the actuators. To the best of our knowledge, the presented methods are mainly based on the backstepping method, which makes this method an appropriate baseline study [25]. To a lesser extent, a nonlinear stochastic system was studied, under the condition of actuator dead-zone, which considers either the time-delay $[17,18]$, or pure-feedback control design method [20]. It is important to note that in most of practical cases, the control systems, i.e., autonomous vehicle systems, nonlinear stochastic conditions are involved [26, 27]. In addition to these conditions, nonlinear behaviors such as dead-zone and hysteresis are typically seen in the actuators [11-19, 21]. Ignoring such the conditions can lead to serious flaws like internal instability and physical damages. However, recently adaptive dynamic surface control for uncertain nonstrict-feedback systems is investigated in [28, 29].

In this paper, neural network in conjunction with dynamic surface control design is employed to introduce a novel method of adaptive control design for nonlinear stochastic systems with a general class of different actuator nonlinearities, including PI hysteresis and dead-zone. These nonlinearities might be a result of actuator aging, a faulty condition of the actuator, or its intrinsical characteristic. The unknown dynamics of the system are innovatively approximated using a Radial Basis Function (RBF) neural network, where the universal approximation capability of the method makes it possible to approximate a wide range of nonlinear Lipschitz functions. Furthermore, the minimal-learning-parameters algorithm is elaboratively employed to reduce the number of adaptive parameters in an online updating way, which effectively reduces the calculational complexities. In order to show effectiveness of the RBF in both the parameter approximation, and in the nonlinearity compensation of the actuators, a sophistication of the method is also proposed as a baseline method for comparison. In this baseline, compensation of the actuator nonlinearity is performed using an adaptive eliminating term.

The stability analysis of the proposed method along with the baseline are theoretically proved and confirmed by simulation. Performance of the direct method of backstepping is also investigated as another baseline for comparison. It is shown that the proposed controller guarantees the boundedness of all the closed-loop signals, where the tracking error remains in an arbitrary small vicinity of the origin, in terms of the mean quartic value. It is shown that the proposed method exhibits superior performance both in the failure-free condition and in different cases of the actuator nonlinearity, compared to the baselines.

The presented method offers extensive applications in a broad range of the engineering and industrial fields such as flight control [30], autonomous vehicle control systems [31, 32], turbo-machine design [33-35], piezo-actuators [36] and micro-electro-mechanical-systems (MEMSs) [37], and also in various military applications [38].

The main contributions of the paper are: (1) presenting a novel neural network-based method for designing adaptive controller for nonlinear stochastic system with broad range of the actuator nonlinearity, (2) presenting a sophistication of the method as a baseline for the study, in which nonlinearity of the actuator is directly compensated without using the neural network, (3) analytically proving stability of the mentioned methods in failure free condition and also at the presence of the actuator nonlinearities, i.e., PI hysteresis, and dead-zone, (4) exploring performance of the direct backstepping method, detailed in [12], for a broad range of the actuator nonlinearity, as the second baseline study, (5) comparing the proposed method along with the two baselines using different cases of actuator nonlinearities, and studying privileges and limitations of each of the methods.

The paper is organized as follows. Section 1, presents a literature review on the previously published studies. In Section 3, preliminaries and problem statements are described. In Section 4, the methods along with the theorems are presented, which contains the main contributions of the paper. Simulation examples are presented in Section 5. In Section 6 and 7, discussion and conclusion of the paper are presented, respectively. In addition to the main sections of the paper, there are also five appendices, in which details of the theorem proofs are included accordingly.

\section{Related studies}

Actuator failure can occur in many practical systems, named plants, that may lead to the plant instability and even sometime catastrophic events [1-7, 27, 39-44]. Systematic design methods for different nonlinear control systems have been studied in the form of the strict-feedback, pure feedback, and block-strict-feedback [45], where various direct methods have been investigated for the purpose of actuator failure compensation [39-44]. Backstepping design method was proposed as a systematic adaptive controller design, which is still considered as one of the mostly used methods for nonlinear systems. Backstepping-based methods for compensation of the actuator failures such as sliding-mode control [42], and adaptive failure compensation [5, 39, 41, 43, 44, 46-49] have been proposed for several practical and theoretical systems. Among these methods, the problem of accommodating infinite number of actuator failures/faults in control systems has been investigated in [5]. Backstepping method 
was theoretically studied to be employed for adaptive control design for the parameter-strict-feedback systems [43], and its capabilities in compensating actuator nonlinearities for a flight control system were investigated [11]. Radial Bases Function (RBF) neural network has been integrated with backstepping method to overcome the problem of uncertain nonlinear systems in pure-feedback form with PI hysteresis [21]. Backstepping controller design method using adaptive neural networks was proposed in conjunction with variable separation and minimallearning-parameters algorithm technique for stochastic nonlinear single-input-single-output systems in the form of nonstrict-feedback with unknown backlash-like hysteresis [21], strict-feedback [20], and pure-feedback [50], [51].

Although the backstepping design technique has many useful benefits for the designers, it suffers from an inherent problem, called 'explosion of complexity', that occurs with increasing system order, due to the continuously differentiation of virtual control signal and system states. Dynamic Surface Control (DSC) method was introduced as another alternative method, which resolves explosion of complexity [8-10, 23, 32, 36-38, 52, 53]. It avoids continuous differentiation of virtual control inputs leading to 'explosion of terms'. DSC has been privileged over backstepping in several studies [9]. Integration of adaptive neural network and DSC was studied in nonlinear strict-feedback systems [12], and also in time-delayed nonlinear systems [15], under failure-free condition of the actuators, as well as a certain form of the PI hysteresis [16]. The effect of actuator dead-zone in nonlinear systems was separately studied for adaptive DSC method [23].

Application of dynamic surface method has been studied in several applied researches, such as controlling pneumatic servo system [32], trajectory tracking control of underactuated surface vehicles [36], suppressing chatter in a micro-milling machine with piezo-actuators [37], controlling micro-electro mechanical gyroscope systems [38], controlling process of continuous heavy cargo airdrop of nonlinear transport aircraft [52], controlling of spacecraft terminal safe approach with actuator saturation [53], and precise position tracking problem of permanent magnet synchronous motors [54].

In many practical systems their parameters, and dynamics, as well as the corresponding disturbances are unknown, but can likely show stochastic and mostly nonlinear characteristics. Details of a complete course of stochastic systems and stochastic differential equation are found in [17], [18].

Adaptive neural networks were employed in conjunction with the dynamic surface technique for nonlinear stochastic systems with either time-delays or dead-zone in the actuators [15]. A certain class of nonlinear systems, but not stochastic, with unknown Prandtl-Ishlinskii hysteresis was studied by $X$. Zhang et al. and the performance of the design method was investigated [22]. In this study, an adaptive neural DSC controller was constructed to eliminate the effect of unknown actuator hysteresis. The adaptive neural network was utilized in DSC design method to stabilize nonlinear time-delay systems with unknown disturbances [9]. Adaptive neural network control systems have been investigated for specific cases of uncertain nonlinear strict-feedback systems [12], and also a class of time-delay nonlinear systems with PI hysteresis with dynamic uncertainties [16-18]. Nevertheless, for nonlinear stochastic cases, the adaptive neural network dynamic surface design was studied only under the condition of time delayed and actuator dead-zone [25].

\section{Preliminaries and problem statement}

A stochastic nonlinear system with strict-feedback can be defined by its state variable $x=\left[x_{1}, x_{2}, \ldots, x_{n}\right]^{T} \in R^{n}$ :

$$
\left\{\begin{array}{c}
d x_{1}=\left(g_{1} x_{2}+f_{1}\right) d t+\psi_{1} d \psi \\
\vdots \\
d x_{i}=\left(g_{i} x_{i+1}+f_{i}\right) d t+\psi_{i} d \psi b_{m} \leq g_{i} \leq b_{M} \\
\vdots 1 \leq i \leq n \\
d x_{n}=\left(g_{n} u+f_{n}\right) d t+\psi_{n} d \psi, \\
y=x_{1},
\end{array}\right.
$$

where $\psi$ is an $r$-dimensional variable introduced as standard Brownian motion defined on a complete probability space, ${ }^{1}$ and $f_{i}(\cdot), g_{i}(\cdot): R^{i} \times R^{+} \rightarrow R, \psi_{i}^{T}: R^{i} \times R^{+} \rightarrow R^{i \times r}$ are unknown smooth functions in $x_{i} \in R^{i}$ with zero initial conditions [25]. It should be noted that $u$ in Eq. (1) is the control input that is by itself the output of an actuator, which can be subjected to different nonlinearities such as Prandtl-Ishlinskii (PI) hysteresis, or dead-zone.

Prandtl-Ishlinskii (PI) hysteresis is a nonlinearity defined as follows:

$u(t)=p_{0} v(t)-\int_{0}^{R} p(r) F_{r}[v](t) d r$

where $u(t)$ is the output of the actuator, $v(t)$ is the input signal to the actuator, $p(r)$ is the density function, $p_{0}=$ $\int_{0}^{R} p(r) d r$ is a constant which depends on the density function $p(r)$, and $F_{r}[v](t)$ is a function, describing the nonlinearity behavior, and named the "play operator" [13]. It should be noted that Eq. (2) decomposes the hysteretic

\footnotetext{
1 The comprehensive details of Brownian motions and complete probability space can be found in [54].
} 
action into two terms, describing the linear reversible part and the nonlinear hysteretic behavior, at its first and the second terms, respectively. This decomposition is crucial since it facilitates utilization of the currently available control techniques for the controller design [15]. An actuator with PI hysteresis is a component with memory, and therefore its value depends on its previous outputs in time. Consequently, for an input $v(t) \in C_{m}\left[0, t_{E}\right]$, where $C_{m}\left[0, t_{E}\right]$ is the space of piecewise monotone continuous functions, and the play operator is defined by:

$$
\begin{aligned}
& F_{r}\left[v ; u_{-1}\right](0)=f_{r}\left(v(0), u_{-1}\right) \\
& F_{r}\left[v ; u_{-1}\right](t)=f_{r}\left(v(t), F_{r}\left[v ; u_{-1}\right]\left(t_{i}\right)\right), \\
& \text { for } t_{i}<t \leq t_{i+1} \text { and } 0 \leq i \leq N-1,
\end{aligned}
$$

with

$f_{r}(v, u)=\max (v-r, \min (v+r, u))$

where $0=t_{0}<t_{1}<\ldots<t_{N}=t_{E}$ is a partition of $\left[0, t_{E}\right]$ such that the function $v$ is monotone on each of the sub-interval $\left[t_{i}, t_{i+1}\right]$, and $u_{-1} \in R$ is the general initial condition [13].

Consider the PI-model expressed by the play operator in (7), the hysteresis output $u(t)$ can be expressed as [14]:

$u(t)=p_{0} v(t)-d[v](t)$

where

$d[v](t)=\int_{0}^{R} p(r) F_{r}[v](t) d r$, with $p_{0}=\int_{0}^{R} p(r) d r$

It should be noted that (11) is bounded, and the detailed description of its boundedness is discussed in [16-20]. Furthermore, in this paper it is assumed that the characteristics of PI hysteresis nonlinearity in the actuator is unknown and should be estimated by the controller.

Actuator dead-zone is another form of the nonlinearity model can be described as follows:

$u=D(v)=\left\{\begin{array}{l}g_{r}(v), v \leq b_{l} \\ 0, b_{l}<v<b_{r} \\ g_{r}(v), v \geq b_{r}\end{array}\right.$

where $u$ is the output of the dead-zone, $v$ is the input of the dead-zone, $b_{l}<0$ and $b_{r}>0$ are unknown parameters of the dead-zone, which should be estimated by the control system, named as the start and end of the dead-zone, respectively. The output of the dead-zone is not measurable, and therefore the smooth and bounded first derivative functions $g_{l}(v)$ and $g_{r}(v)$ are employed to express the output. In order to achieve a pseudolinear relationship between the input and output of the dead-zone, the following expression is often employed:

$u(t)=D(v)=K^{T}(t) \Phi(t) v+d(v)$ where detailed description of functions $K^{T}(t), \Phi(t)$, and $d(v)$ can be found in [16]-[19]. However, $K^{T}(t) \Phi(t)$ is bounded, $|d(v)| \leq p^{*}$, and $p^{*}$ is an unknown positive constant [19].

One way to approximate the unknown dynamic of the actuator nonlinearity is the use of a Radial Basis Function Neural Network (RBF). It provides universal approximating capability, by which any unknown continuous function $f(Z): R^{n} \rightarrow R$ can be approximated as follows:

$f(Z)=W^{* T} \zeta^{T}(Z)+\delta(Z)$

where $Z \in \Omega_{\mathrm{Z}} \subset R^{q}$ is the input vector with $q$ being the neural networks input dimension, $W=\left[w_{1}, w_{2}, \cdots, w_{l}\right]^{T} \in R^{l}$, is the weight vector of neural networks with $l>1$, the neural networks node number, and $\zeta(Z)=\left[\varsigma_{1}(Z), \varsigma_{2}(Z), \cdots, \varsigma_{l}(Z)\right]$ is the basis function vector with $\varsigma_{i}(Z)$ being chosen as Gaussian function following the form:

$\varsigma_{i}(Z)=\exp \left(-\frac{\left(Z-\mu_{i}\right)^{T}\left(Z-\mu_{i}\right)}{\eta_{i}^{2}}\right), i=1, \cdots, l$

The $\mu_{i}=\left[\mu_{i 1}, \cdots, \mu_{i q}\right]$ is the center of the respective field and $\mu_{i}$ is the width of the Gaussian function [25]. $\delta(Z)$ is the approximation error and satisfies $|\delta(Z)| \leq \varepsilon, \varepsilon>0$. $W^{* T}$ is the ideal constant weight vector [25] and is defined as:

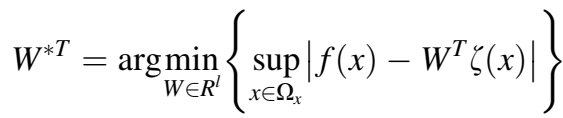

For simplicity, by using the minimal-learning-parameters algorithm an unknown constant $\theta$ is introduced as:

$\theta=\max \left\{\frac{1}{b_{m}}\left\|W_{j}^{* T}\right\| ; j=1,2, \ldots, n\right\}$

We consider a stochastic nonlinear system in strictfeedback form with unknown dynamics where the actuator is subjected to a nonlinearity. The method proposed by the following sequels employs a radial basis neural network to estimate unknown dynamics of the system, and hence to design the adaptive control method.

\section{Methods}

\subsection{Overview}

The proposed control design method is based on using the dynamic surface as a systematic controller design in conjunction with an adaptive RBF neural network to serve as a global approximator meant for unknown dynamics, nonlinearities, and stochastic behaviors of the system. The 
method which we call Adaptive Neural Network Dynamic Surface Control (ANNDSC) is independently investigated for nonlinear stochastic strict-feedback systems using three different actuator characteristics: linear, nonlinear with dead-zone, and nonlinear with hysteresis characteristics. The probability boundedness of all the closed-loop signals will be proven via stability analysis in an analytic way, and the simulations support the theories for all the three cases of the actuator nonlinearity.

In order to demonstrate effectiveness of RBFNN in compensating actuator nonlinearity, a modification of the ANNDSC method is introduced as a baseline for comparison. This baseline method is named Adaptive PI Compensation using Dynamic Surface Control (APIC-DSC), where the adaptive term is employed to directly compensate the PI hysteresis nonlinearity. The stability analysis of the last method is also analytically proven. In order to show effectiveness of both the ANNDSC and the APIC-DSC, compared to the existing design method, another baseline is defined based on the direct implementation of the backstepping design method. Technical details of the design method for this baseline are found in [25]. For further clarity, and meanwhile maintaining continuity of the subjects, proofs of the presented theorems are included in the appendices.

\subsection{The proposed method ANNDSC}

Consider a nonlinear stochastic strict-feedback system defined by Eq. (1), ANNDSC offers an iterative procedure with $n$ steps ( $n$ is order of the system) for designing the control system. At each step of the method, the error surface is firstly calculated by subtracting state variables from the desired output. Then, the calculated error is passed through a first-order filter, for all the steps, but the last step. An RBF neural network employs the filtered error to approximate dynamic of the system. The error surface $\left\{S_{i}\right\}$, for step $i, \quad 1 \leq i \leq n$, is defined as:

$S_{i}=x_{i}-z_{i}, 1 \leq i \leq n$,

where $x_{i}$ and $z_{i}$ are the corresponding state variable and desired state value, respectively. For $i=1, z_{1}=y_{r}$, where $y_{r}$ is the reference input, the desired output of the system. The proposed procedure involves $n$ successive steps of computation, as depicted in Fig. 1.

A virtual control input $\bar{x}_{i+1}$ is defined at each step:

$$
\begin{aligned}
\bar{x}_{i+1} & =-k_{i} S_{i}-\frac{1}{2 a_{i}} S_{i}^{3} \hat{\theta} \zeta_{i}^{T}\left(Z_{i}\right) \zeta_{i}\left(Z_{i}\right), 1 \leq i \leq n, Z_{i} \\
& =\left[\breve{x}_{i}, \hat{\theta}\right], \breve{x}_{i}=\left[x_{1}, \ldots, x_{i}\right],
\end{aligned}
$$

$v=\bar{x}_{n+1}=-k_{n} S_{n}-\frac{1}{2 a_{n}} S_{n}^{3} \hat{\theta} \zeta_{n}^{T}\left(Z_{n}\right) \zeta_{n}\left(Z_{n}\right)$,

where the $a_{i}, k_{i}$ are the design parameters, $\zeta_{i}\left(Z_{i}\right)$ are the radial basis functions of the corresponding neural network, and $\widehat{\theta}$ is an estimation of $\theta$. The virtual control input is passed through a low-pass filter to obtain the desired value for the next state:

$\epsilon_{i+1} \dot{z}_{i+1}+z_{i+1}=\overline{\mathrm{x}}_{i+1}, 1 \leq i \leq n-1$

where the $\epsilon_{i+1}, 1 \leq i \leq n-1$ are the design constants. Finally, the RBF neural network weights are approximated using the following expression:

$\dot{\hat{\theta}}=\sum_{j=1}^{n} \frac{\lambda}{2 a_{j}^{2}} S_{j}^{6} \zeta_{j}^{T}\left(Z_{j}\right) \zeta_{j}\left(Z_{j}\right)-k_{0} \widehat{\theta}$

where $\lambda$ is a design constant, and $\zeta_{j}(\cdot), 1 \leq j \leq n$ are the basis functions of the neural network. The RBF neural network is indeed composed of two layers. The first layer incorporates $l$ nodes. Each node $i(1 \leq i \leq l)$ corresponds to a Gaussian function of center $\eta_{i}$, and width $\mu_{i}$. The three parameters $\left(l, \eta_{i}, \mu_{i}\right)$ are treated as the design parameters. The second layer is a linear superposition of the Gaussian functions, using the learning weight $W$. Norm of the learning weights is employed for the approximation.

Theorem 1 Applying the ANNDSC controller design method, to a nonlinear stochastic strict-feedback system with a linear actuator and any unknown dynamics, Eq. (1), guarantees the boundedness in probability of all closedloop signals of the system.

Proof 1 The comprehensive proof of this theorem is explained in Appendix 2.

Theorem 2 Applying the ANNDSC controller design method, to nonlinear stochastic strict-feedback systems with any unknown dynamics, Eq. (1), which is subjected to a hysteresis nonlinearity in its actuator, guarantees that all the closed-loop signals of the system remain bounded in probability.

Proof 2 The comprehensive proof of this theorem is explained in Appendix 4.

Theorem 3 Applying the ANNDSC controller design method, to a nonlinear stochastic system in strict-feedback form with any unknown dynamics, Eq. (1), subjected to actuator dead-zone nonlinearity, guarantees the boundedness in probability of all closed-loop signals of the system.

Proof 3 The comprehensive proof of this theorem is explained in Appendix 5. 
Fig. 1 Flowchart of the proposed method, ANNDSC, for nonlinear stochastic systems in the form of strict-feedback

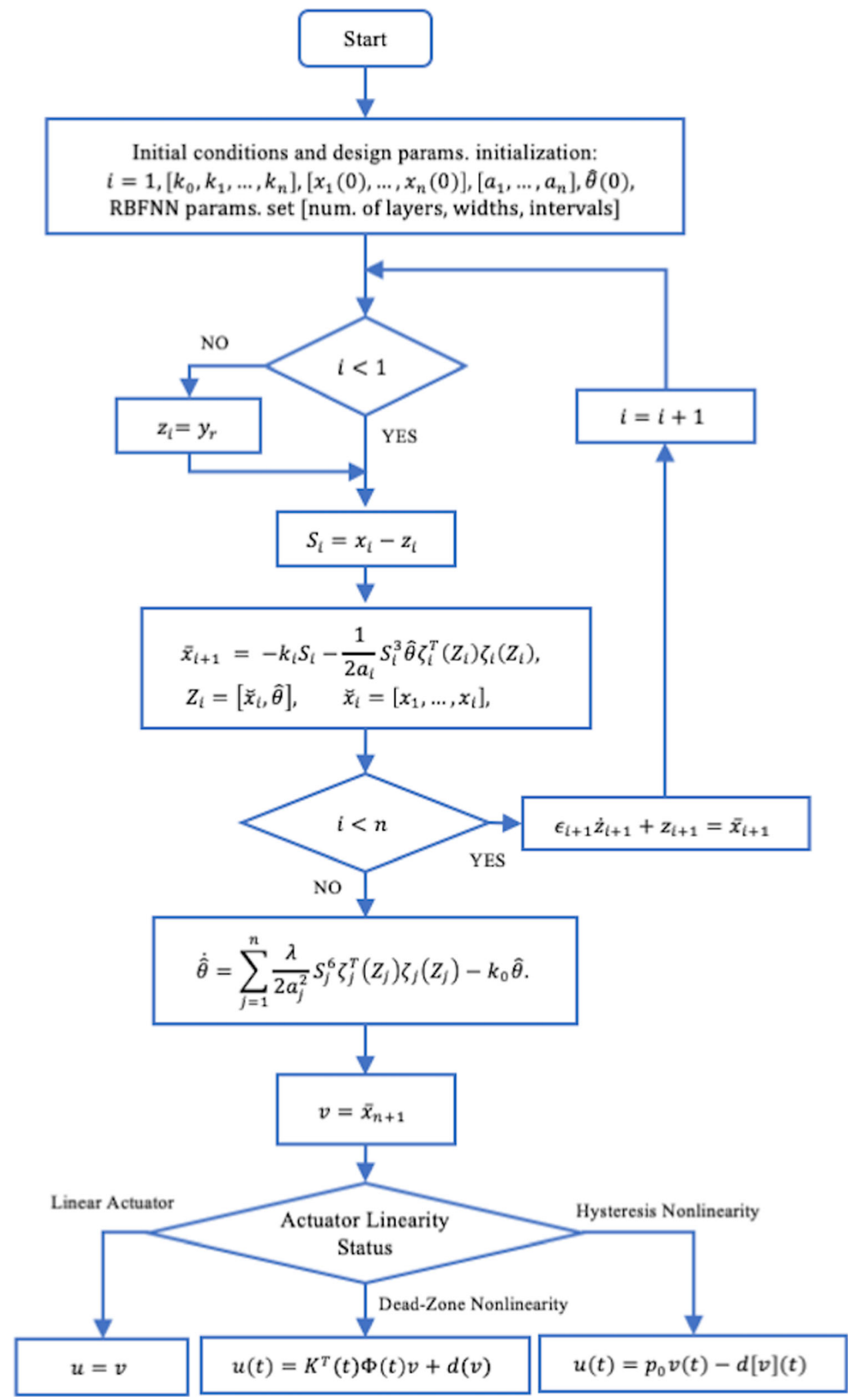

Experimentation of ANNDSC is herein described through a practical example of a hypersonic aircraft cruising at a Mach number of 15 and an altitude of 110,000 $\mathrm{ft}$, which is subjected to a nonlinear stochastic condition. The control system comprises two separate controllers, for the velocity and the flight path angle [24]. Dynamic of the 
flight path angle can be expressed by the state equation system (Eq. 18) using three state variables: $x=\left[x_{1}, x_{2}, x_{3}\right]^{T}=[\gamma, \theta, q]^{T}:$

$$
\begin{array}{r}
d x_{1}=\left(f_{1}\left(x_{1}\right)+g_{1}\left(x_{1}\right) x_{2}\right) d t+\psi_{1} d \psi \\
d x_{2}=x_{3} d t+\psi_{2} d \psi \\
d x_{3}=\left(f_{3}\left(x_{1}, x_{2}, x_{3}\right)+g_{3}\left(x_{1}, x_{2}\right) u\right) d t+\psi_{3} d \psi \\
y=x_{1}
\end{array}
$$

The three state variables $\gamma, \theta$ and $q$ are the flight path angle, the altitude, and the pitch rate, respectively. The functions $\boldsymbol{f}_{1}\left(\boldsymbol{x}_{1}\right), \boldsymbol{f}_{3}\left(\boldsymbol{x}_{1}, \boldsymbol{x}_{2}, \boldsymbol{x}_{3}\right), \boldsymbol{g}_{1}\left(\boldsymbol{x}_{1}\right)$, and $\boldsymbol{g}_{3}\left(\boldsymbol{x}_{1}, \boldsymbol{x}_{2}\right)$ are the nonlinear functions describing dynamic of the system. The $\psi_{i}, 1 \leq i \leq 3$ are unknown smooth functions, and $\mu$ is a constant number. Details of finding dynamic model of the system are found in [24]. The system defined in Eq. (18) demonstrates dynamics of a nonlinear stochastic strictfeedback plant. The flight path $\boldsymbol{y}$ can be controlled using the ANNDSC.

\subsection{Adaptive PI compensation DSC (APIC-DSC)}

In ANNDSC, the proposed neural network provided sufficient tools both for the control design and for compensating the actuator nonlinearity. The proposed baseline of APIC-DSC is introduced to investigate effect of using neural network for compensating the actuator nonlinearity, proposed by ANNDSC. In this baseline study, an adaptive PI hysteresis compensator is proposed using direct method, in conjunction with the adaptive RBF neural network to compensate actuator nonlinearity of kind Prandtl-Ishlinskii (PI) hysteresis, in contrast with ANNDSC in which the neural network undertook the compensation task. as defined in Eqs. (2) to (5). In this situation, for each step $i$, $i \leq i \leq n$, the error surface and the virtual control inputs are defined as in Eq. (13) and Eq. (14). Figure 2 illustrates flowchart of the method.

The virtual control input is passed through a low-pass filter to obtain the desired value for the next state:

$\epsilon_{i+1} \dot{z}_{i+1}+z_{i+1}=\overline{\mathrm{x}}_{i+1}, 1 \leq i \leq n-1$

where $\epsilon_{i+1}, 1 \leq i \leq n-1$ are design parameters. The control input is:

$$
\begin{aligned}
v & =-\overline{\mathrm{x}}_{n+1} \\
& =-k_{n} S_{n}-\frac{1}{2 a_{n}^{2}} S_{n}^{3} \widehat{\theta} \zeta_{n}^{T}\left(Z_{n}\right) \zeta\left(Z_{n}\right)+\int_{0}^{R} \widehat{p}_{p_{0}}(t, r)\left|F_{r}[v](t)\right| d r
\end{aligned}
$$

where the $a_{i}, k_{i}$ are design parameters, $\zeta_{i}\left(Z_{i}\right)$ are the radial basis functions, $\widehat{\theta}$ and $\widehat{p}_{p_{0}}$ are the estimations of $\theta$ and $p_{p_{0}}$, respectively. The $\widehat{p}_{p_{0}}$ is approximated using an adaptive law:

$$
\frac{\partial}{\partial t} \widehat{p}_{p_{0}}(t, r)=\left\{\begin{array}{c}
-\gamma_{p r}\left[\left|S_{n}^{3}\right|\left|F_{r}[u](t)\right|+\sigma \widehat{p}_{p_{0}}(t, r)\right], 0 \leq \widehat{p}_{p_{0}} \leq p_{\max }, \\
-\sigma \widehat{p}_{p_{0}}(t, r), \widehat{p}_{p_{0}}>p_{\max },
\end{array}\right.
$$

where the $\sigma$ and $p_{\max }$ are positive design parameters, $p_{p_{0}}(t, r)=p(t, r) / p_{0}$, and $p_{p_{\max }}:=\left(p_{\max } / p_{0}\right)$. Finally, the RBF weights are approximated using the following adaptive law:

$\dot{\hat{\theta}}=\sum_{j=1}^{n} \frac{\lambda}{2 a_{j}^{2}} S_{j}^{6} \zeta_{j}^{T}\left(Z_{j}\right) \zeta_{j}\left(Z_{j}\right)-k_{0} \widehat{\theta}$

where $\lambda$ is a design constant, and $\zeta_{j}(\cdot), 1 \leq j \leq n$ are the basis functions of the neural network.

It can be seen from (22) and (21) that both the nonlinearities and the system dynamics are approximated using the adaptive law in Eq. (22) resulted from the neural network weights; however, the density function of the PI integral is directly approximated using the adaptive law in Eq. (21).

Theorem 4 Applying the APIC-DSC controller design method, to a nonlinear stochastic system with any unknown dynamics, subjected to actuator PI hysteresis nonlinearity guarantees the boundedness in probability of all closedloop signals of the system.

Proof 4 The comprehensive proof of this theorem is explained in Appendix 6.

\section{Simulation results}

Performance of the proposed ANNDSC method, along with the two baseline methods, is evaluated and compared in a tracking problem using a 3rd-order benchmark system. Details of the benchmark system are found in [25]. Another alternative benchmark of 2rd-order system can be found in [25], but we used the 3rd-order ones with more complexities to explore performance of the methods and hence provide a better comparison, under a rather complex condition. This benchmark for study considers a stochastic nonlinear system in strict-feedback form: 
Fig. 2 Flowchart of the baseline method, named APIC-DSC, for nonlinear stochastic systems in strict-feedback form

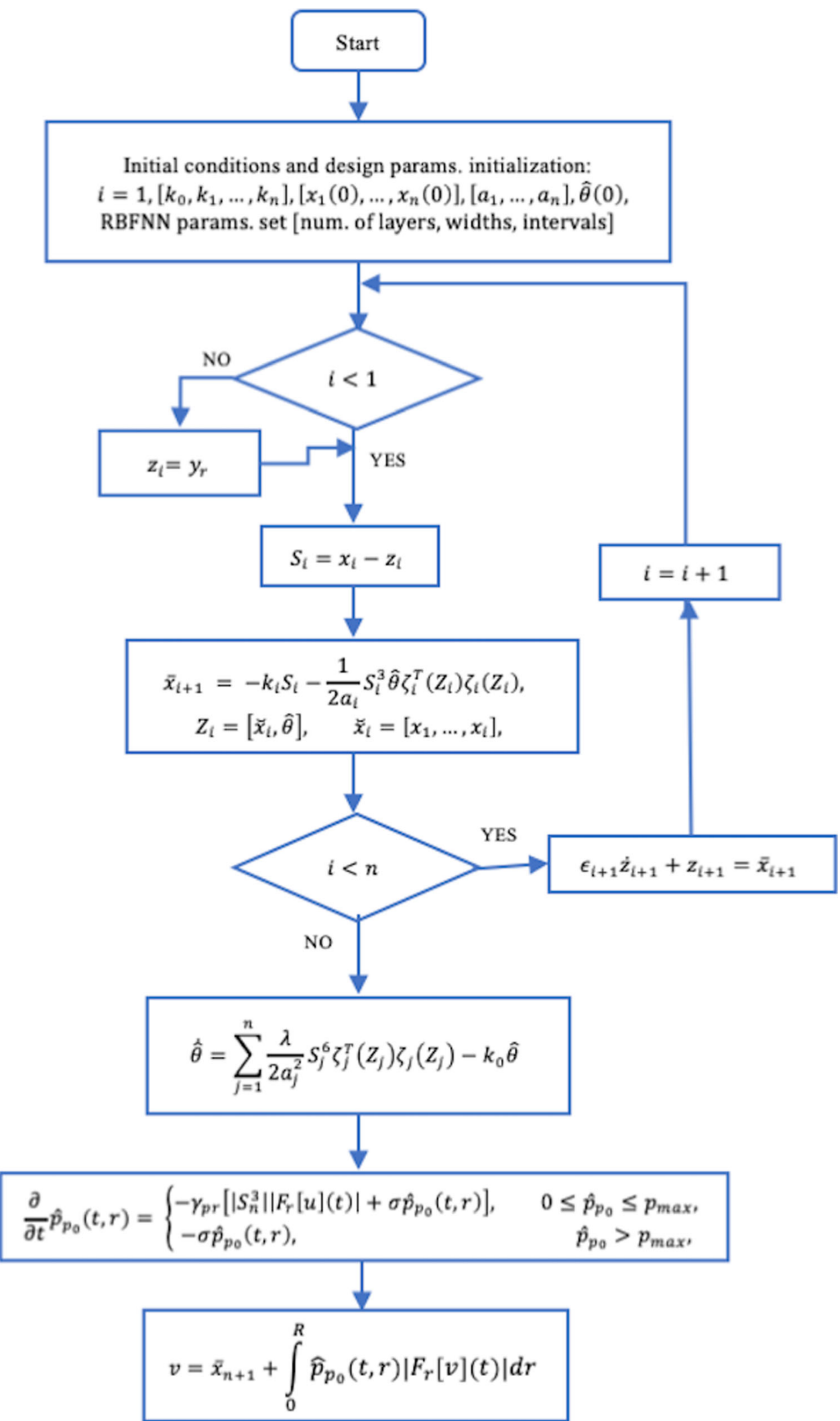


$d x_{1}=\left(\left(0.3+x_{1}^{2}\right) x_{2}-0.8 \sin \left(x_{1}\right)\right) d t+x_{1} \sin \left(x_{1}\right) d \omega$,

$d x_{2}=\left(\left(1+x_{2}^{2}\right) x_{3}-x_{2}-0.5 x_{2}^{3}-x_{1}^{3}-\sqrt{x_{1}}\right) d t+x_{1} \cos \left(x_{2}\right) d \omega$,

$d x_{3}=\left(\left(1.5+\sin \left(x_{1} x_{2}\right)\right) u-0.5 x_{3}-\frac{1}{3} x_{3}^{2}-x_{2}^{2} x_{3}-\frac{x_{1}}{\left(1+x_{1}^{2}\right)}\right) d t$

$+3 x_{1} e^{-x_{2}^{2}} d \omega$

$y=x_{1}$

$y_{r}=\sin (t)$

The simulation study considers a failure-free condition together with two other cases of the actuator nonlinearity, i.e., actuator dead-zone, and the actuator PI hysteresis for the proposed method, ANNDSC, along with the two baseline design methods, named APIC-DSC and backstepping design, respectively. The ANNDSC-based controller for the failure-free, and the two cases of nonlinearity, is designed using Eqs. (13)-(17) as follows:

$$
\left\{\begin{array}{l}
S_{1}=x_{1}-y_{r} \\
\overline{\mathrm{x}} 2=-k_{1} S_{1}-\frac{1}{2 a_{1}^{2}} S_{1}^{3} \widehat{\theta} \zeta_{1}^{T}\left(Z_{1}\right) \zeta_{1}\left(Z_{1}\right) \\
\epsilon_{2} \dot{z}_{2}+z_{2}=\overline{\mathrm{x}} 2 \\
S_{2}=x_{2}-z_{2} \\
\overline{\mathrm{x}} 3=-k_{2} S_{2}-\frac{1}{2 a_{2}^{2}} S_{2}^{3} \widehat{\theta} \zeta_{2}^{T}\left(Z_{2}\right) \zeta_{2}\left(Z_{2}\right) \\
\epsilon_{3} \dot{z}_{3}+z_{3}=\overline{\mathrm{x}}_{3} \\
S_{3}=x_{3}-z_{3}, \\
v=-k_{3} S_{3}-\frac{1}{2 a_{3}^{2}} S_{3}^{3} \widehat{\theta} \zeta_{3}^{T}\left(Z_{3}\right) \zeta_{3}\left(Z_{3}\right) \\
\dot{\hat{\theta}}=\sum_{k=1}^{3} \frac{\lambda}{2 a_{k}^{6}} S_{k}^{6} \zeta_{k}^{T}\left(Z_{k}\right) \zeta_{k}\left(Z_{k}\right)-k_{0} \widehat{\theta}
\end{array}\right.
$$

where $Z_{1}=S_{1}, Z_{2}=\left[S_{1}, S_{2}, \widehat{\theta}\right], Z_{3}=\left[S_{1}, S_{2}, S_{3}, \widehat{\theta}\right], p(r)=$ $e^{-0.067(r-1)^{2}}, r \in[0,10], v(t)=\frac{7 \sin (3 t)}{1+t}, t \in[0,2 \pi], \quad$ and $u_{-1}=0$ are the input vectors of neural networks. Equation (24) shows the design steps of a control system of order 3, which clearly involves three design steps. At each of the step, firstly the error surface $S_{i}$ is calculated by subtracting the desired value $z_{i}$ from the actual value of state $x_{i}$. Using the error surface, along with the neural network weights, $\widehat{\theta}$, the virtual control input of the step, $x_{i+1}$, is estimated. The desired value is calculated for each step by passing the virtual control input signal through a first-order filter, except for the final step, where the actual control input is directly generated. Subsequently, the neural network weights are found using the adaptive law, by which the unknown dynamics and nonlinearities of the system will be approximated.

The design parameter set for the simulation is $\left[k_{0}, k_{1}, k_{2}, k_{3}\right], \quad\left[a_{1}, a_{2}, a_{3}\right], \epsilon_{2}=0.006$, and $\epsilon_{3}=0.008$, which are obtained empirically considering the transient performance, the limitations on control effort growth, the closed-loop internal stability of the system, and improvement in the tracking error. The simulation runs under the initial condition of $\left[x_{1}(0), x_{2}(0), x_{3}(0)\right]^{T}=[0,0.6,0.4]^{T}, \widehat{\theta}(0)=0.1$. The three RBF neural networks $W_{1}^{T} \zeta_{1}\left(Z_{1}\right), W_{2}^{T} \zeta_{2}\left(Z_{2}\right)$, and $W_{3}^{T} \zeta_{3}\left(Z_{3}\right)$ are chosen to contain eleven nodes with the centers spaced evenly within the intervals $[-5,5]$, $[-5,5] \times[-5,5] \times[-5,5], \quad$ and $[-5,5] \times[-5,5] \times[-5,5] \times[-5,5]$, respectively.

The APIC-DSC baseline controller for the system in Eq. (23) is designed using Eqs. (13), (14), (19)-(22). This controller is completely similar to Eq. (24), unless the controller input signal:

$$
\begin{aligned}
v= & -k_{3} S_{3}-\frac{1}{2 a_{3}^{2}} S_{3}^{3} \widehat{\theta}_{3}^{T}\left(Z_{3}\right) \zeta_{3}\left(Z_{3}\right) \\
& +\int_{0}^{R} \widehat{p}_{p_{0}}(t, r)\left|F_{r}[v](t)\right| d r,
\end{aligned}
$$

$\frac{\partial}{\partial t} \widehat{p}_{p_{0}}(t, r)=\left\{\begin{array}{c}-\gamma_{p r} S_{n}^{3} F_{r}[u](t)-\gamma_{p r} \sigma \widehat{p}_{p_{0}}(t, r), \widehat{p}_{p_{0}}>p_{\max } \\ -\gamma_{p r} \sigma \widehat{p}_{p_{0}}(t, r), \widehat{p}_{p_{0}}<p_{\max }\end{array}\right.$

where $\left[k_{0}, k_{1}, k_{2}, k_{3}\right],\left[a_{1}, a_{2}, a_{3}\right]$ are design parameters, obtained empirically likewise the ANNDSC case. $p_{\text {max }}=0.2, R=2, \gamma_{p r}=1, \sigma=2$, and other parameters are similar to the previous simulation step. The backstepping case is entirely implemented as detailed in [25].

Simulation results of these three cases are depicted in Figs. 3, 4, 5, 6 and 7. The system output is depicted against the reference sinusoidal input, for all the three methods, under the three actuator conditions.

Minimal deflection from the desired form of sinusoidal wave is seen for all the methods and conditions. In order to quantitate the deflection from the desired output, the Integrated Mean Square Error of the actual outputs is calculated with respect to the inputs. Figure 4 demonstrates the IMSE for the all methods and conditions.

Outperformance of ANNDSC is seen for all the case with the minimal IMSE. In the tracking problem, lower IMSE is regarded as an indication of the better performance. For the failure free cases, the relative depression in IMSE of the proposed method is observed to be $25 \%$ and $11 \%$ as compared to the backstepping and APIC-DSC, respectively. Nevertheless, effectiveness of the method is further highlighted when there is a nonlinearity condition in the actuator. For the PI hysteresis, the proposed method improves the tracking performance by $76 \%$ and $38 \%$ as reflected by the relative IMSE for the backstepping and APIC-DSC method, respectively. For the dead-zone nonlinearity, this relative outperformance is, however, $32 \%$ and $49 \%$, showing a good improvement in the tracking performance. For the dead-zone condition, the APIC-DSC 

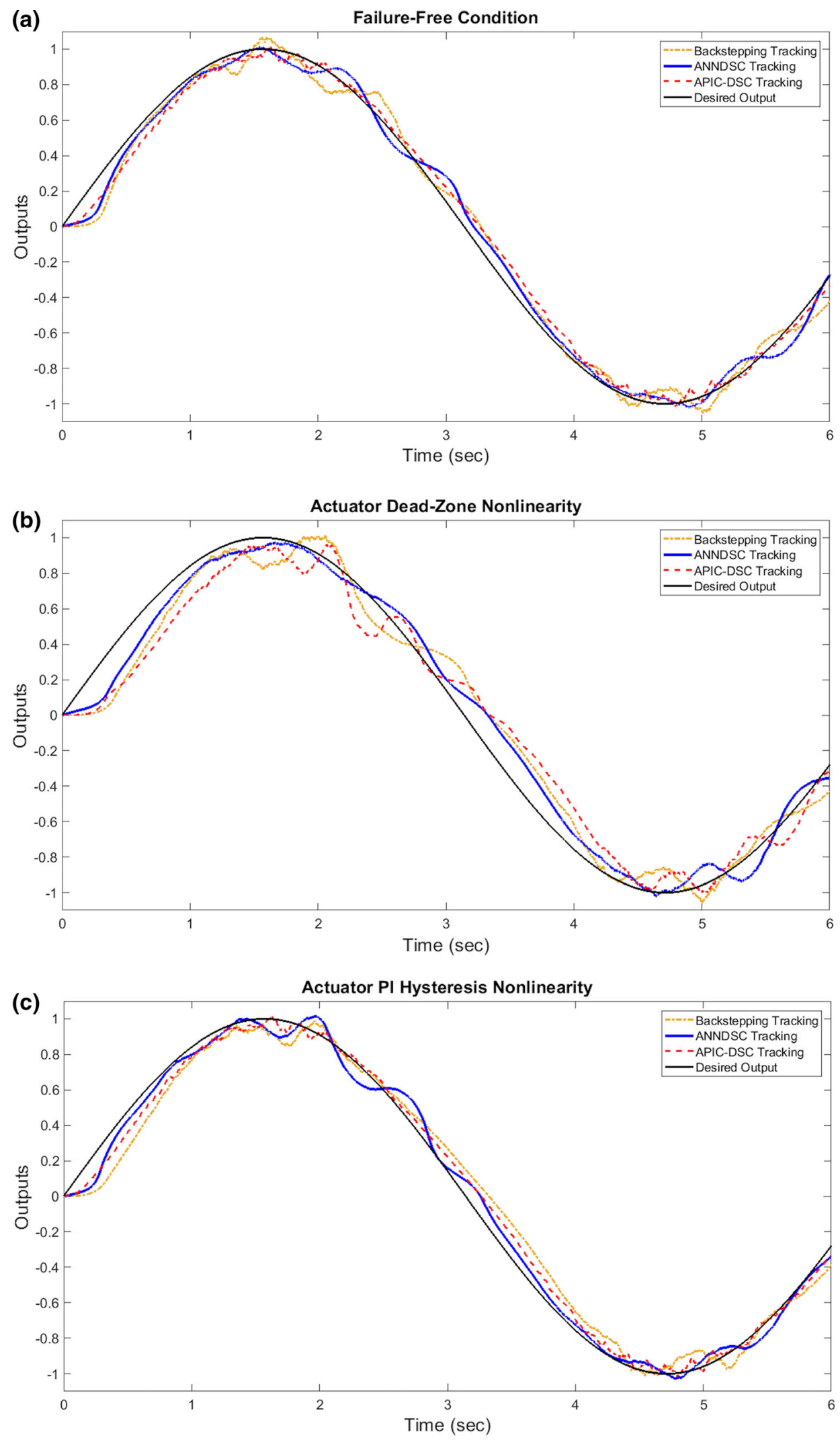

Fig. 3 Output of the three control systems and the reference input, for three different characteristics of the actuators 
offers the worst IMSE, implying that improvement in the PI hysteresis is served at the expense of impairing the performance for other condition, when direct method is employed. It is also seen that all the methods offer their optimal performance at the absence of the actuator nonlinearity.

In order to investigate internal stability of the control methods, closed-loop signals and states of the three control systems are plotted with different actuator nonlinearities. Figure 5 shows the closed-loop states.

The close-loop signals of the APIC-DSC are considerably higher than the ANNDSC and the backstepping, showing further tendency to internal instability in practical situations, even though the values are bounded. This is confirmed by the control input signal, depicted in Fig. 6.

The ANNDSC method exhibits smaller control effort, compared to the two baselines. The control input signal of ANNDSC shows smoother and low oscillatory waveform, which provides a more reliable functionality in practice. The risk of the internal stability is by far highest for the APIC-DSC, even though the outputs are not far different for all the methods. It is important to note that high amplitude of the control input signal can practically put the system into the risk of actuator saturation. These conditions sometimes make finding a control strategy impractical, despite showing acceptable tracking.

Figure 7 demonstrates adaptive law of the three methods.

As seen in Fig. 7, the adaptive law, $\widehat{\theta}$, damps quicker for the ANNDSC and APIC-DSC, revealing faster convergence for the neural network-based methods compared to the backstepping one. Figures 8 and 9 show the system outputs and the control inputs, for a case of the joint dead- zone and PI hysteresis nonlinearities, occurring at two different time instances.

All the three methods show good performance in tracking the output. However, the APIC-DSC dramatically increases the control inputs on the occurrence of the deadzone. This makes the APIC-DSC an inappropriate candidate for the practical situations, where such the large value of the control input put the system into the risk of saturation.

\section{Discussion}

The paper suggested an adaptive control design method for nonlinear stochastic systems with a general class of the actuator nonlinearity. In contrast to the existing techniques relying on the backstepping design method [11]-[21], the proposed method employed dynamic surface control design, along with neural networks through an algorithm of minimal learning parameters, to avoid the "explosion of complexity" and decline the computational efforts. This favorable feature which cannot be seen in the backstepping-based methods will become especially important for the systems with increased order. Such the implementation improves agility of the design method to be suitable for an online application. The paper proved boundedness of all the closed-loop signals and convergence of all the error signals to a small vicinity of the origin at the presence of two different nonlinearities, commonly seen at the actuators, dead-zone and hysteresis, in both analytic and simulation manners.

Although certain nonlinearities have been investigated in recent studies [25], the joint dead-zone and hysteresis were not included in the studies. In many practical
Fig. 4 Integrated Mean Square Error (IMSE) of a tracking problem for the proposed method (ANNDSC), the baseline methods 1 (APICDSC), and the baseline method 2 (backstepping), under different conditions of failurefree, actuator dead-zone and Prandtl-Ishlinskii (PI) hysteresis
IMSE

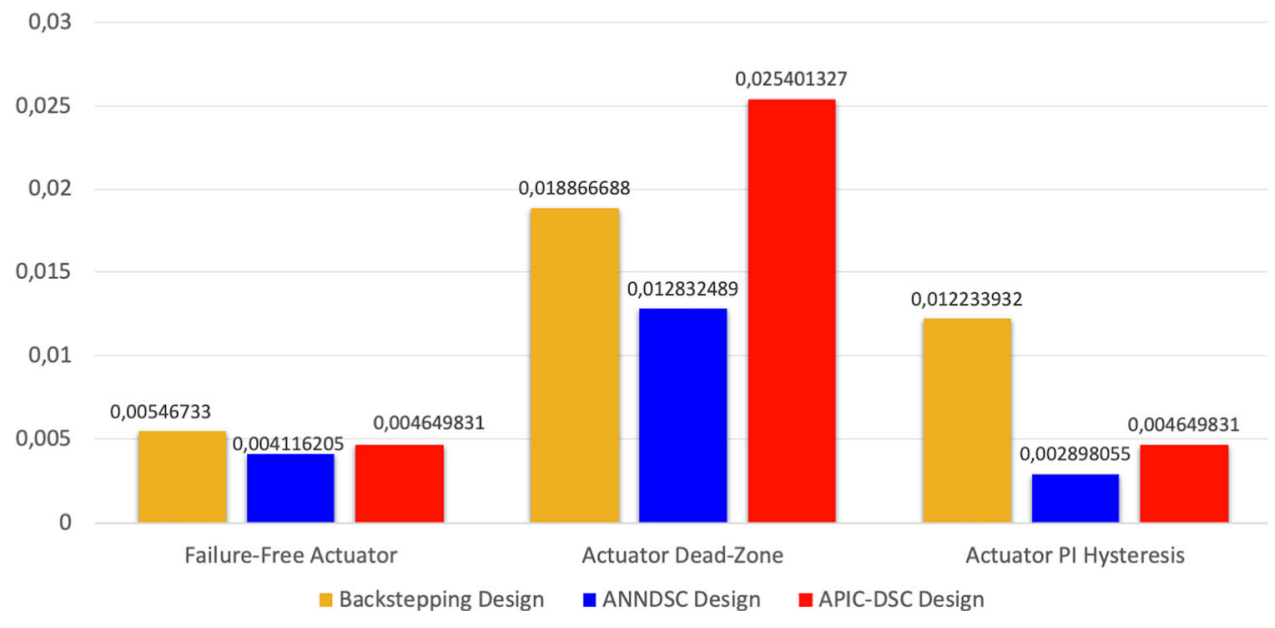


Fig. 5 Comparison of the closed-loop states of the proposed control system (ANNDSC) along with the baseline methods APIC-DSC and backstepping in the tracking problem. The three actuator nonlinearities are separately illustrated for the third order system with the three state variables
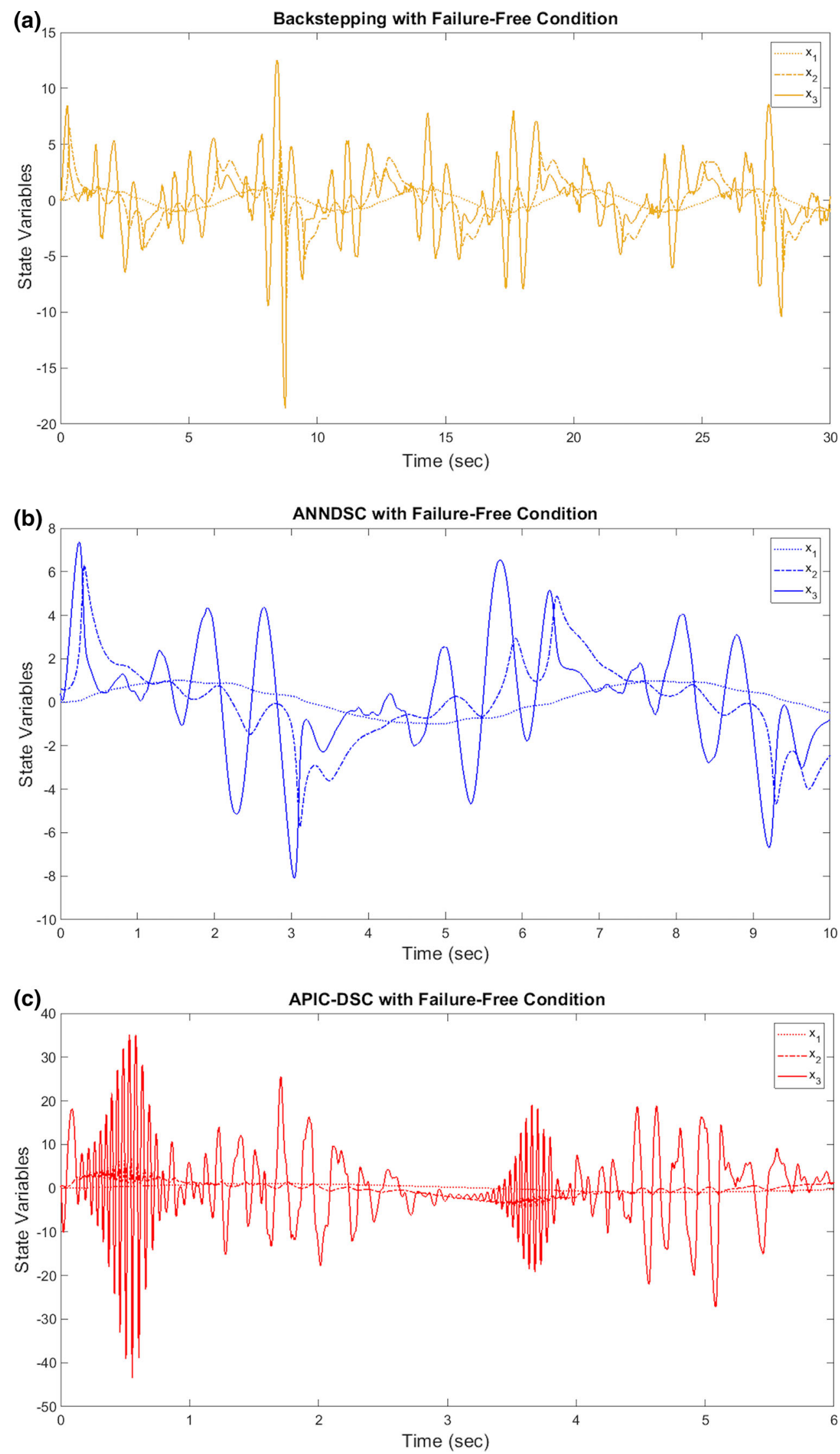
(d)

Backstepping with Actuator Dead-Zone Nonlinearity
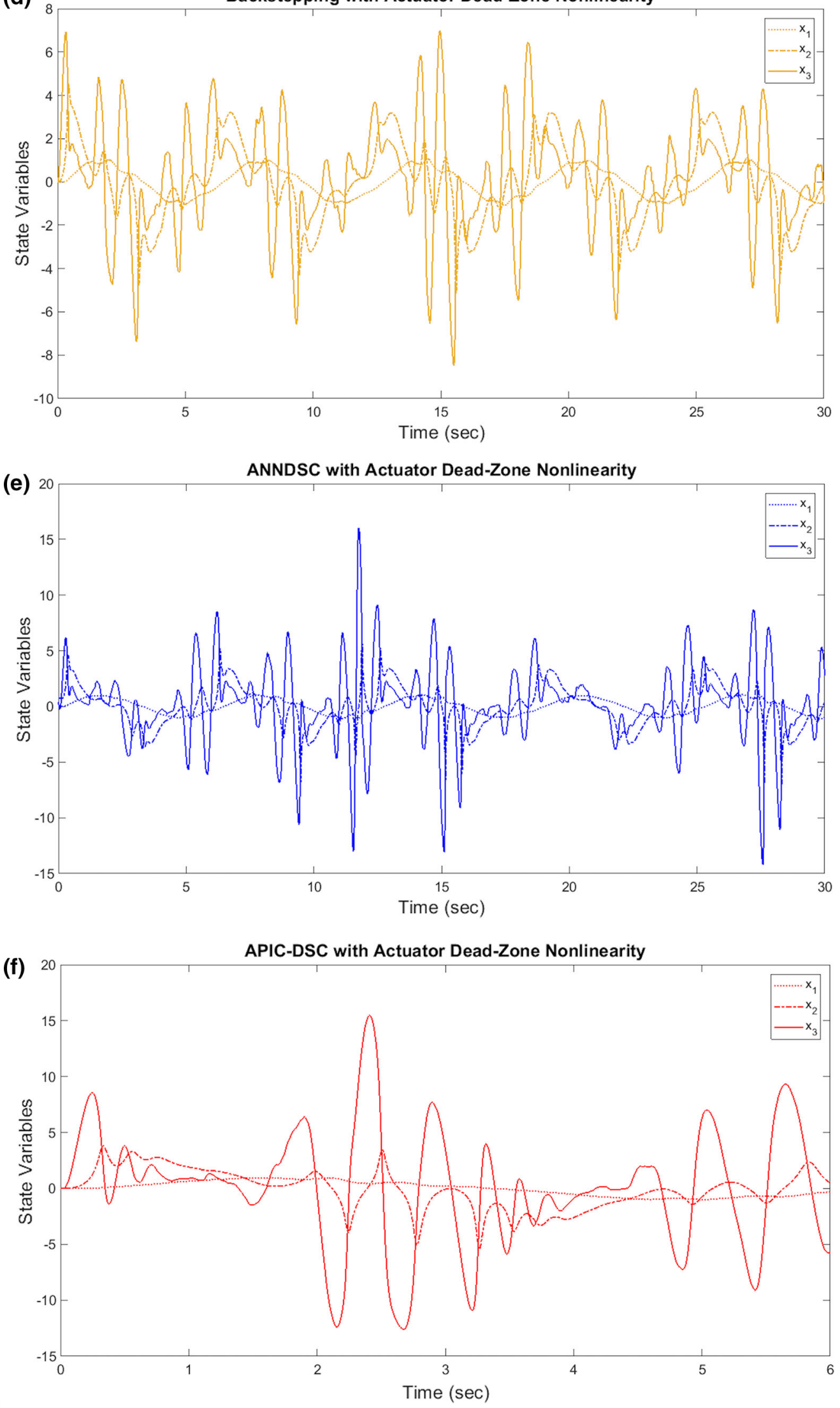

Fig. 5 continued 

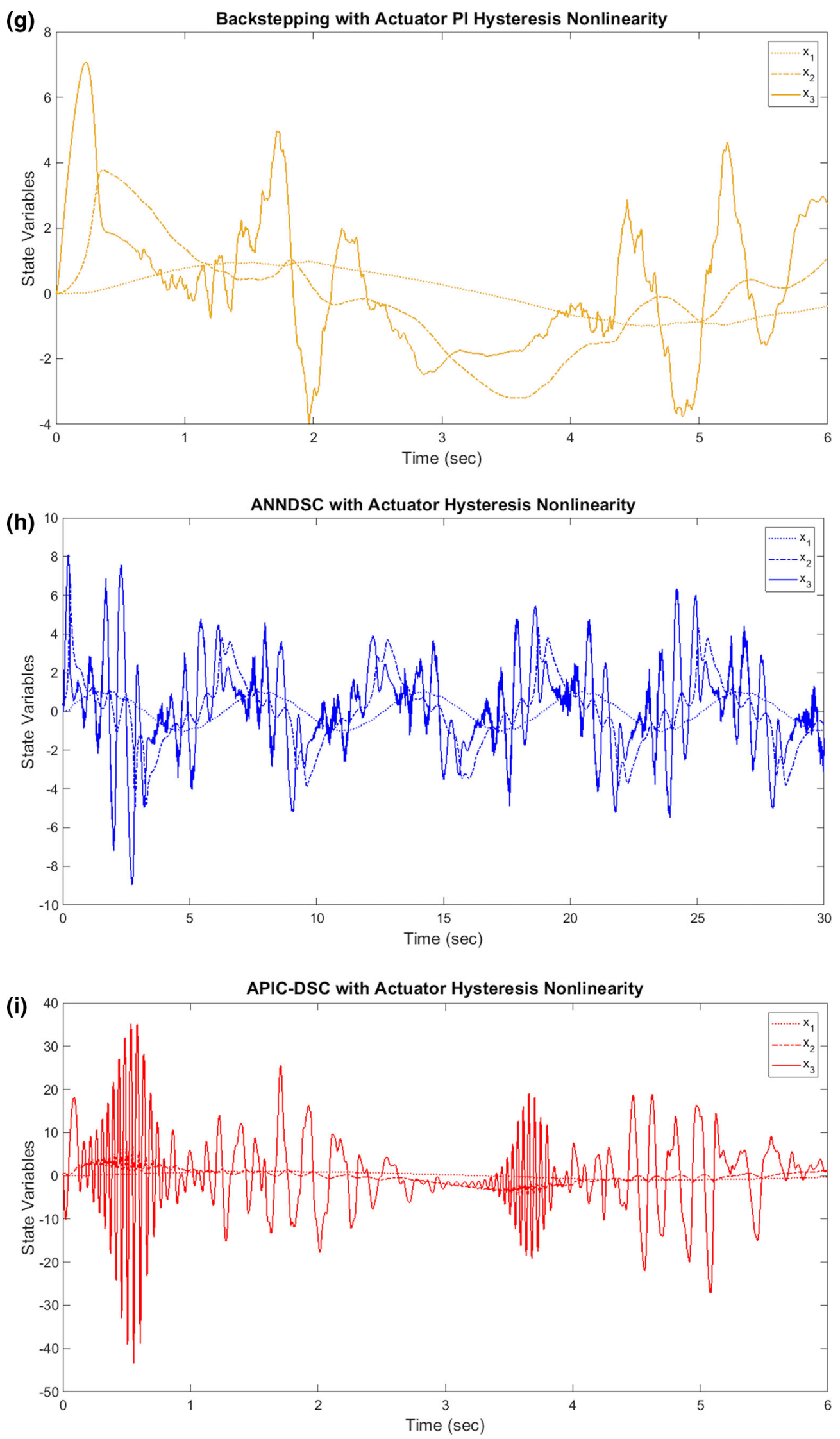

Fig. 5 continued 
(a)

Failure-Free Condition
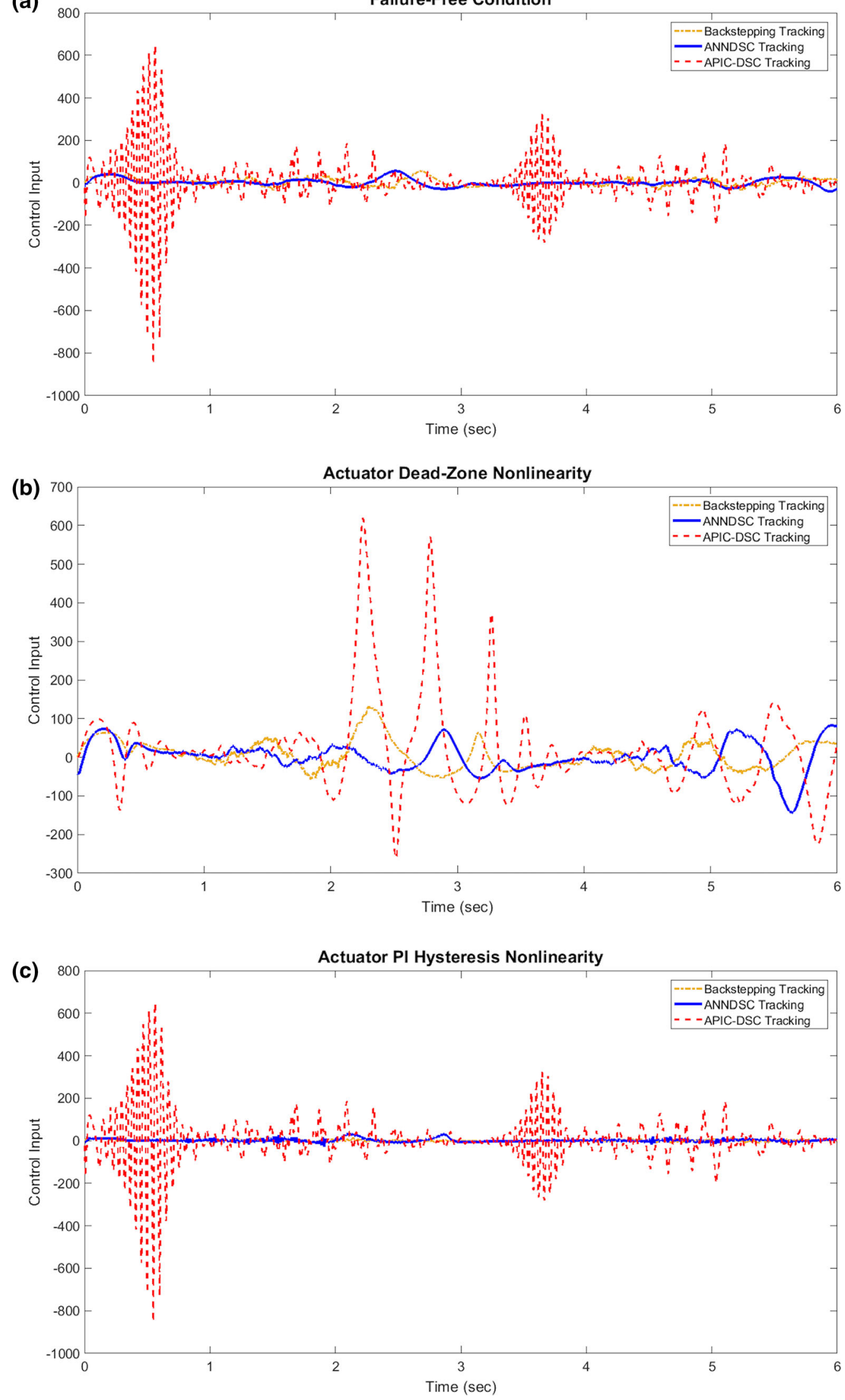

Fig. 6 Control input of the three systems in the tracking problem, described in the sequel 

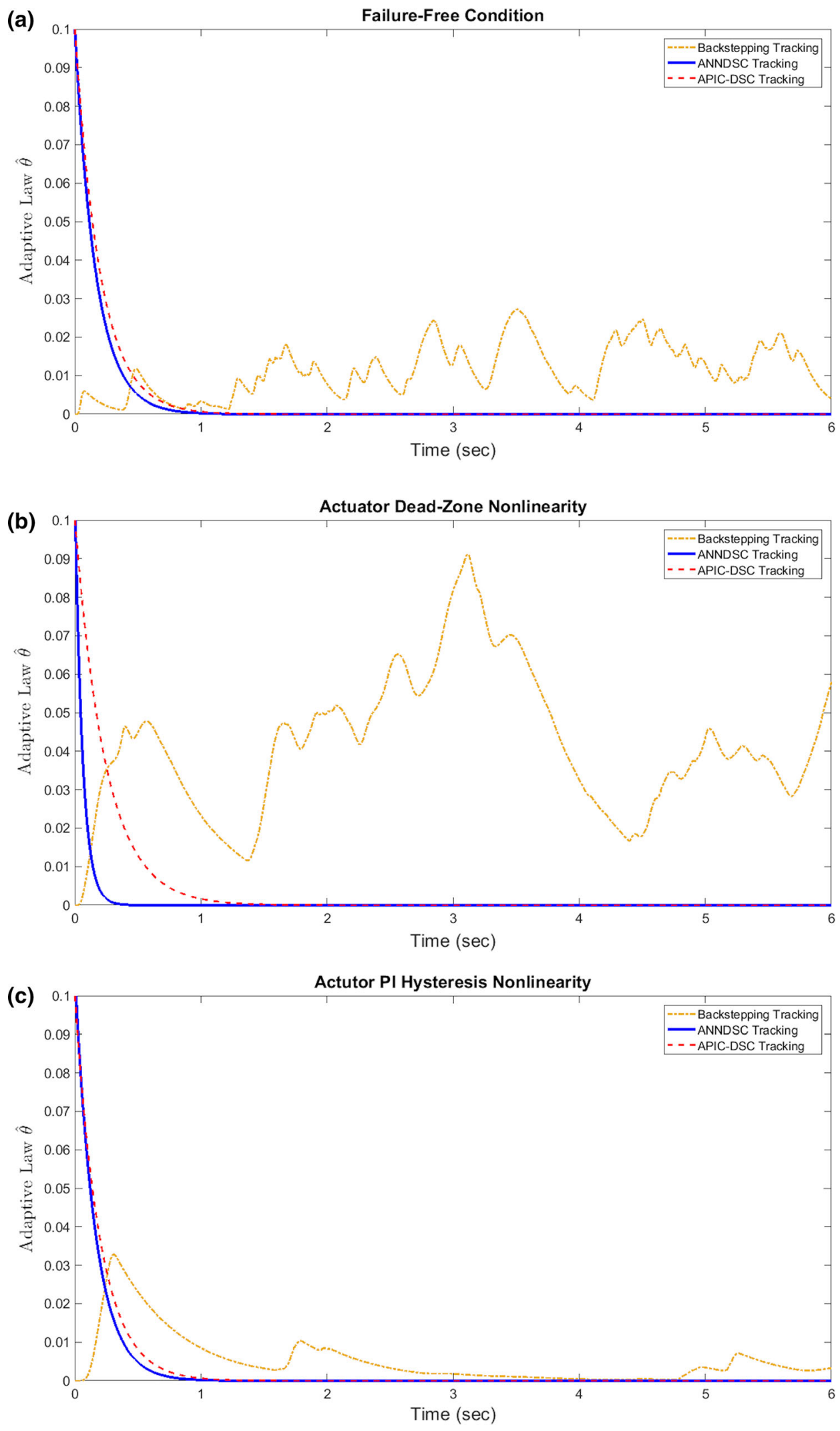

Fig. 7 Adaptive law of the three methods for the tracking problem 
applications, actuators can accidentally encounter with any of the dead-zone and hysteresis, due to the aging. It is sometimes critically important to consider such the conditions in the design method.

We introduced a baseline method for nonlinear stochastic system, named APIC-DSC, sophisticated for compensating the actuator hysteresis. In this baseline method, adaptive neural network is not invoked for the compensation. It is analytically proved that the closed-loop signals remain bounded in probability. This method although shows acceptable performance for the failure-free and also for the hysteresis conditions, but dramatically increases the control input at the presence of the dead-zone.

Considering Figs. 5 and 9, the control effort of the ANNDSC is much less than the two other baseline methods. It is possible to improve the tracking at the cost of increasing the control effort. It might, however, lead to actuator saturation or internal instability of the system. It was observed that the control effort is by far lower for ANNDSC than the two baseline methods.

In this study, the proposed method was empirically optimized by jointly considering the tracking performance and the control effort. Among the design parameters, the set of $\left[k_{0}, k_{1}, k_{2}, k_{3}\right]$ and $\left[a_{1}, a_{2}, a_{3}\right]$ have more effect on the transient and the steady state characteristics of the system where the $k_{3}$, and $a_{3}$ directly affect the control input of the system. However, the proposed method can be well-integrated with the genetic algorithm for finding an optimal set of the design parameters. This is also true for other metaheuristic methods, or natural-based algorithms, such as ant colony algorithm. For our baseline study of backstepping method, we used the same set of the design parameters described in [25] as an initial set, and followed similar
Fig. 8 Output of the three control systems and the reference input, for the joint dead-zone and hysteresis nonlinearity, occurring at the seconds 1 and 4

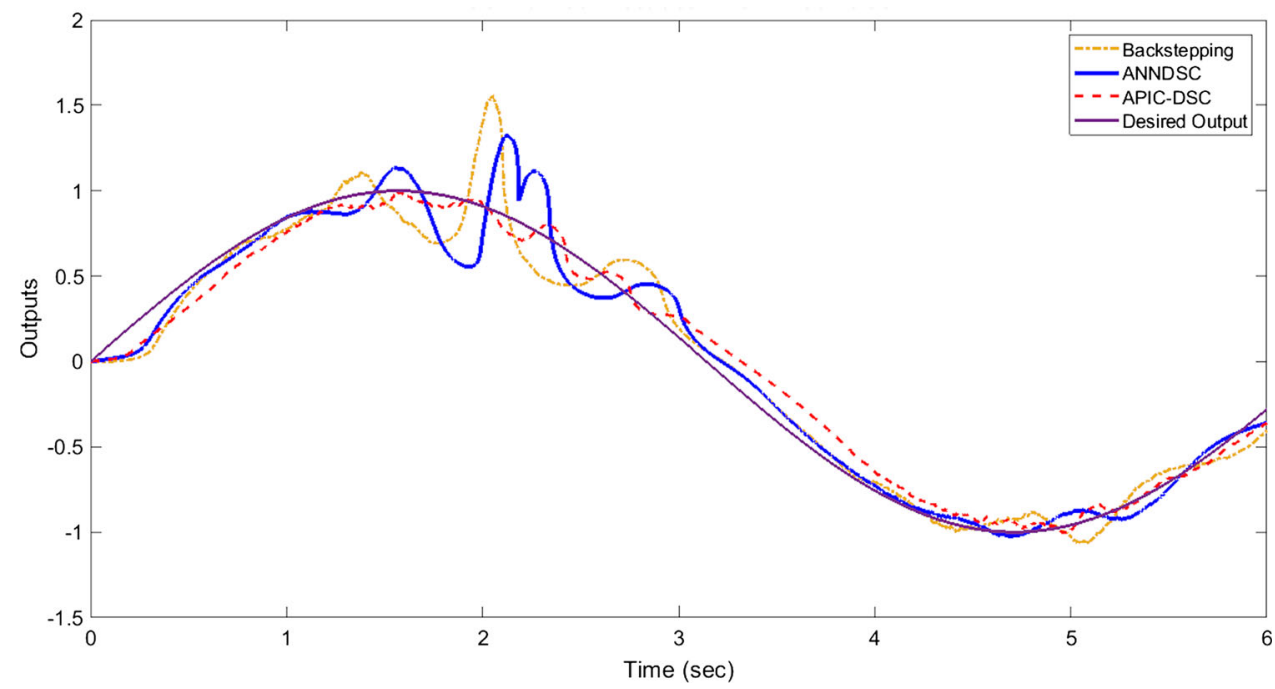

Fig. 9 Control input of the three systems in the tracking problem, for the joint dead-zone and hysteresis nonlinearity, occurring at the seconds 1 and 4

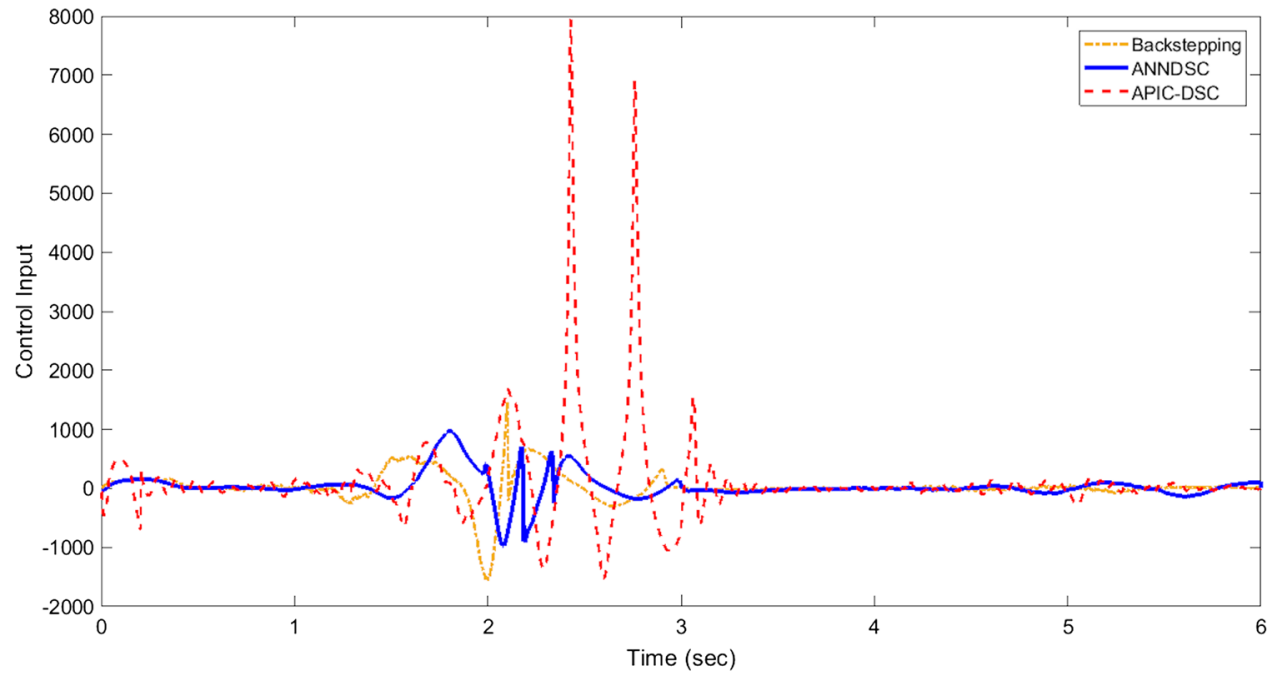


empirical procedure for improving the performance, as was done for ANNDSC and APIC-DSC.

Selecting an appropriate sampling rate plays an important role in efficient performance of any control system. A low sampling rate can lead to system instability, while on the other hand, an excessive sampling rate increases redundant complexities. A recent study proposed an interesting systematic method, named FIRCEP, that can be easily employed for finding an optimal sampling rate [55].

We used MATLAB R2017b for the simulations and analysis. Nowadays, there are various platforms, commercially available for efficient implementation in the practical situations and real plants, such as PLC systems with strong computational power. It is obvious that such the implementations demand a level of the practical considerations.

\section{Conclusion}

This paper proposed a novel adaptive design method for nonlinear stochastic control systems using neural network. The proposed method was investigated under joint conditions of the actuator nonlinearities, defined as the deadzone and the Prandtl-Ishlinskii hysteresis. Stability analysis was analytically studied and confirmed by the simulation results in a tracking problem. Performance of the proposed method was compared to a baseline of widely used method, the backstepping method. It is observed that using the proposed neural network in conjunction with the dynamic surface method, considerably enhances performance of the control design method, and meanwhile decreases the computational complexities as well as the control effort.

\section{Appendix 1}

Consider the following stochastic system:

$d x=f(x, t) d t+h(x, t) d \psi$

where $x=\left[x_{1}, x_{2}, \ldots, x_{n}\right]^{T} \in R^{n}, \psi$ is an $r$-dimensional standard Brownian motion defined on the complete probability space $(\Omega, F, P)$, and $\Omega$ is a sample space, $F$ is a $\sigma$ field, $\left\{F_{t}\right\}_{t \geq 0}$ is a filtration, $P$ is a probability measure, and $f: R^{n} \times R^{+} \rightarrow R^{n}, \quad h: R^{n} \times R^{+} \rightarrow R^{n \times r} \quad$ are locally Lipschitz functions $\quad$ in $x \in R^{n}$, with $f(0, t)=0, h(0, t)=0, \forall t \geq 0$.

Definition 1 Wang et al. [25] For any given $V(x, t) \in$ $C^{2,1}\left(R^{n} \times R^{+} ; R^{+}\right)$, associated with the stochastic differential Eq. (26), we define the differential operator $L$ as follows:
$L V=\frac{\partial V}{\partial t}+\frac{\partial V}{\partial x} f+\frac{1}{2} \operatorname{Tr}\left\{h^{T} \frac{\partial^{2} V}{\partial x^{2}} h\right\}$

Remark 1 The term $\frac{1}{2} \operatorname{Tr}\left\{h^{T} \frac{\partial^{2} V}{\partial x^{2}} h\right\}$ is called Itô correction term, in which the term $\frac{\partial^{2} V}{\partial x^{2}}$ introduces a high level of complexity to the controller design procedure in comparison with the deterministic case [25].

Lemma 1 Wang et al. [25] Consider the stochastic system (Eq. 26) and assume that $f(x, t)$, and $h(x, t)$ are $C^{1}$ in their arguments and $f(0, t)$, and $h(0, t)$ are bounded uniformly in $t$. If there exist functions $V(x, t) \in C^{2,1}\left(R^{n} \times R^{+}, R^{+}\right), \quad \mu_{1}(\bullet), \mu_{2}(\bullet) \in K_{\infty}, \quad$ constants $a_{0}>0, b_{0} \geq 0$, such that.

$\mu_{1}(|x|) \leq V(x, t) \leq \mu_{2}(|x|), L V \leq a_{0} V(x, t)+b_{0}$

then the solution process of Eq. (26) is bounded in probability.

Lemma 2 Young's Inequality:

$x y \leq \frac{\alpha^{p}}{p}|x|^{p}+\frac{1}{q \alpha^{q}}|y|^{q},(p-1)(q-1)=1, \alpha>0$

where the constants $p, q$, and $\alpha$ are chosen properly depending on the circumstances [25].

Lemma 3 Wang et al. [25] For any continuous function $f(x): R^{n} \rightarrow$ $R$ with $f(0)=0, x=\left[x_{1}, x_{2}, \ldots, x_{n}\right]^{T}$, there exist positive smooth functions $h_{j}\left(x_{j}\right): R \rightarrow R^{+}, j=1,2, \ldots, n$, such that.

$|f(x)| \leq \sum_{j=1}^{n}\left|x_{j}\right| h_{j}\left(x_{j}\right)$

\section{Appendix 2}

A consistent technique is used to arrive at the conclusions in Eq. (45), Eq. (62), and Eq. (46), which is described in the following sequel. The aim is to approve the following equation:

$\begin{aligned} g_{i} S_{i}^{3} h_{i}\left(Z_{i}\right) & \leq \frac{b_{m}}{2 a_{i}^{2}} S_{i}^{6} \theta \zeta_{i}^{T}\left(Z_{i}\right) \zeta_{i}\left(Z_{i}\right)+\frac{1}{2} a_{i}^{2} b_{M}^{2}+\frac{3}{4} g_{i}^{\frac{4}{3}} S_{i}^{4} \\ +\frac{1}{4} \varepsilon_{i}^{4}, 1 & \leq i \leq n\end{aligned}$

Using the expanded expression of $h_{i}\left(Z_{i}\right)$ in Eq. (63), for the $g_{i} S_{i}^{3} h_{i}\left(Z_{i}\right)$ term in Eq. (63), $1 \leq i \leq n$ it can be written as: 


$$
\begin{aligned}
g_{i} S_{i}^{3} h_{i}\left(Z_{i}\right) & =g_{i} S_{i}^{3}\left(W_{i}^{* T} \zeta_{i}\left(Z_{i}\right)+\delta_{i}\left(Z_{i}\right)\right) \\
& =\underbrace{g_{i} S_{i}^{3} W_{i}^{* T}\left(\frac{W_{i}^{*}}{W_{i}^{*}}\right) \zeta_{i}\left(Z_{i}\right)}_{\text {firstterm }}+\underbrace{g_{i} S_{i}^{3} \delta_{i}\left(Z_{i}\right)}_{\text {seconderm }}
\end{aligned}
$$

For the first term in Eq. (32) using the Young's inequality (4) with the corresponding parameters $x=S_{i} \frac{W_{i}^{* T}}{\left\|W_{i}^{*}\right\|}\left\|W_{i}^{*}\right\| \zeta_{i}\left(Z_{i}\right), y=g_{i}, p=q=2, \alpha=a_{i}$, and for second term in Eq. (32) with the corresponding parameters $x=S_{i}^{3} g_{i}, y=\delta_{i}\left(Z_{i}\right), p=4 / 3, q=1, \alpha=1,\left|\delta_{i}\left(Z_{i}\right)\right| \leq \varepsilon_{\mathrm{i}}$, respectively, it yields:

$$
\begin{aligned}
g_{i} S_{i}^{3} h_{i}\left(Z_{i}\right) & \leq \frac{S_{i}^{6}}{2 a_{i}^{2}} \underbrace{\frac{W_{i}^{* T} W_{i}^{*}}{W_{i}^{* 2}}}_{=1} W_{i}^{* 2} \zeta_{i}^{T}\left(Z_{i}\right) \zeta_{i}\left(Z_{i}\right) \\
+ & \frac{1}{2} a_{i}^{2} \underbrace{g_{i}^{2}}_{\leq b_{M}^{2}}+\frac{3}{4} g_{i}^{\frac{4}{3}} S_{i}^{4}+\frac{1}{4} \varepsilon_{i}^{4} \leq S_{i}^{6} \frac{b_{m}}{2 a_{i}^{2}} \underbrace{\frac{W_{i}^{* 2}}{b_{m}}}_{\leq \theta} \zeta_{i}^{T}\left(Z_{i}\right) \zeta_{i}\left(Z_{i}\right) \\
+ & \frac{1}{2} a_{i}^{2} b_{M}^{2}+\frac{3}{4} g_{i}^{\frac{4}{3}} S_{i}^{4}+\frac{1}{4} \varepsilon_{i}^{4},
\end{aligned}
$$

where $a_{i}>0$ and $\varepsilon_{i}>0$. Equation (33) in its simplified form can be written as follows:

$$
\begin{aligned}
g_{i} S_{i}^{3} h_{i}\left(Z_{i}\right) & \leq \frac{b_{m}}{2 a_{i}^{2}} S_{i}^{6} \theta \zeta_{i}^{T}\left(Z_{i}\right) \zeta_{i}\left(Z_{i}\right)+\frac{1}{2} a_{i}^{2} b_{M}^{2}+\frac{3}{4} g_{i}^{\frac{4}{3}} S_{i}^{4} \\
+\frac{1}{4} \varepsilon_{i}^{4}, 1 & \leq i \leq n
\end{aligned}
$$

By this expression, Eq. (31) is proved.

\section{Appendix 3}

\section{Proof of the theorem 1 (Stability analysis)}

The proposed controller design method is based on a multistep recursive design algorithm. In this method, the adaptive neural network approach is implemented using the dynamic surface control in conjunction with minimallearning-parameters algorithm in a recursive manner. At the end of each design step, the resulting data are sent to the next design step. The number of design steps is $n$, which is equal to the number of the system order. At the end of $1 \leq i \leq n-1$ steps, a virtual control signal and a first-order filter are generated, which are sent to the next step. Consequently, in the final step, $n$, the actual control signal is generated, which is sent out from the controller to the actuator.

Step 1
Define the first error surface as:

$S_{1}=x_{1}-y_{r}$

$d S_{1}=d x_{1}-\dot{y_{r}} d t=\left(g_{1} x_{2}+f_{1}-\dot{y_{r}}\right) d t+\psi_{1} d \psi$

By using a stochastic Lyapunov function, it is obtained:

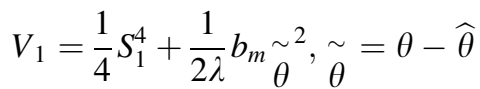

where $\lambda$ is a design constant. Using the Itô's formula, we have $^{2}$ :

$L V_{1}=S_{1}^{3}\left(g_{1} x_{2}+f_{1}-\dot{y}_{r}\right)+\frac{3}{2} S_{1}^{2} \psi_{1}^{T} \psi_{1}-\lambda^{-1} b_{m} \underset{\theta}{\sim} \dot{\hat{\theta}}$

$\frac{3}{2} S_{1}^{2} \psi_{1}^{T} \psi_{1} \leq \frac{3}{2} S_{1}^{4} \varphi_{11}^{2}$

Replacing Eq. (39) in Eq. (38) yields:

$$
\begin{aligned}
L V_{1} & \leq S_{1}^{3}\left(g_{1} x_{2}+f_{1}-\dot{y_{r}}\right)+\frac{3}{2} S_{1}^{4} \varphi_{11}^{2} \\
& -\lambda^{-1} b_{m} \underset{\hat{\theta}}{\sim} \leq S_{1}^{3}\left(g_{1} x_{2}+f_{1}-\dot{y_{r}}+\frac{3}{2} S_{1} \varphi_{11}^{2}\right)+\frac{3}{4} g_{1}^{\frac{4}{3}} S_{1}^{4} \\
& -\frac{3}{4} g_{1}^{\frac{4}{3}} S_{1}^{4}-\lambda^{-1} b_{m} \underset{\theta}{\tilde{\hat{\theta}}}
\end{aligned}
$$

define:

$\overline{\mathrm{f}}_{1} \triangleq f_{1}-\dot{y}_{r}+\frac{3}{2} S_{1} \varphi_{11}^{2}+\frac{3}{4} g_{1}^{\frac{4}{3}} S_{1}$

By adding and subtracting $\frac{3}{4} g_{1}^{\frac{4}{3}} S_{1}$ term to the right-hand side of Eq. (40), and substituting the result in Eq. (41), we have:

$$
\begin{aligned}
\mathrm{LV}_{1} & \leq S_{1}^{3}\left(g_{1} x_{2}+\bar{f}_{1}\right)-\frac{3}{4} g_{1}^{\frac{4}{3}} S_{1}^{4} \\
& -\lambda^{-1} b_{m} \tilde{\theta} \dot{\hat{\theta}} \leq g_{1} S_{1}^{3}(x_{2}+\underbrace{g_{1}^{-1} \bar{f}_{1}}_{\text {unknownterm }})-\frac{3}{4} g_{1}^{\frac{4}{3}} S_{1}^{4} \\
& -\lambda^{-1} b_{m} \tilde{\theta} \dot{\hat{\theta}}
\end{aligned}
$$

Now we approximate the unknown term, $g_{1}^{-1} \bar{f}_{1}$, using a RBFNN [25]. Defining:

$h_{i}\left(Z_{i}\right)=g_{i}^{-1} \mathrm{f}_{i}=W_{i}^{* T} \zeta_{i}\left(Z_{i}\right)+\delta_{i}\left(Z_{i}\right),\left|\delta_{i}\left(Z_{i}\right)\right| \leq \varepsilon_{i}$

where the $Z_{i}=\left[\overline{\bar{x}}_{i}, \hat{\theta}\right], \overline{\bar{x}}=\left[x_{1}, \cdots, x_{i}\right], \quad 1 \leq i \leq n$. Putting $h_{1}\left(Z_{1}\right)$ in the unknown term of Eq. (42) yields:

$L V_{1} \leq g_{1} S_{1}^{3} x_{2}+g_{1} S_{1}^{3} h_{1}\left(Z_{1}\right)-\frac{3}{4} g_{1}^{\frac{4}{3}} S_{1}^{4}-\lambda^{-1} b_{m} \sim \dot{\hat{\theta}}$

Using Eq. (31), the term $S_{1}^{3} g_{1} h_{1}\left(Z_{1}\right)$ can be rewritten as:

\footnotetext{
${ }^{2}$ Using (5), since $\psi_{1}(0)=0$, thus there is a function $\varphi_{11}(\bullet)$ such that $\psi_{1}\left(S_{1}\right)=S_{1} \varphi_{11}\left(S_{1}\right)$
} 


$$
\begin{aligned}
& S_{1}^{3} g_{1} h_{1}\left(Z_{1}\right) \leq \frac{b_{m}}{2 a_{1}^{2}} S_{1}^{6} \theta \zeta_{1}^{T}\left(Z_{1}\right) \zeta_{1}\left(Z_{1}\right)+\frac{1}{2} a_{1}^{2} b_{M}^{2}+\frac{3}{4} g_{1}^{\frac{4}{3}} S_{1}^{4} \\
& \quad+\frac{1}{4} \varepsilon_{1}^{4}
\end{aligned}
$$

and Eq. (44) becomes:

$$
\begin{aligned}
L V_{1} & \leq g_{1} S_{1}^{3} x_{2}+\frac{b_{m}}{2 a_{1}^{2}} S_{1}^{6} \theta \zeta_{1}^{T}\left(Z_{1}\right) \zeta_{1}\left(Z_{1}\right)+\frac{1}{2} a_{1}^{2} b_{M}^{2}+\frac{1}{4} \varepsilon_{1}^{4} \\
& -\lambda^{-1} b_{m} \sim \dot{\hat{\theta}} \leq g_{1} S_{1}^{3} x_{2}+\frac{b_{m}}{2 a_{1}^{2}} S_{1}^{6} \theta \zeta_{1}^{T}\left(Z_{1}\right) \zeta_{1}\left(Z_{1}\right)+\frac{1}{2} a_{1}^{2} b_{M}^{2} \\
& +\frac{1}{4} \varepsilon_{1}^{4}-\lambda^{-1} b_{m} \underset{\theta}{\sim} \dot{\hat{\theta}}
\end{aligned}
$$

By choosing $\overline{\mathrm{x}}_{2}$ as the virtual controller as follows:
Combining Eq. (51) and Eq. (50) and substituting the $x_{2}$ term in Eq. (47) by the resulting, along with mathematical simplification yields:

$$
\begin{aligned}
& L V_{1} \leq-k_{1} g_{1} S_{1}^{4}+g_{1} S_{1}^{3}\left(S_{2}+y_{2}\right) \\
& \quad+\frac{b_{m}}{\lambda} \tilde{\theta}\left(\frac{\lambda}{2 a_{1}^{2}} S_{1}^{6} \zeta_{1}^{T}\left(Z_{1}\right) \zeta_{1}\left(Z_{1}\right)-\dot{\hat{\theta}}\right)+\frac{1}{2} a_{1}^{2} b_{M}^{2}+\frac{1}{4} \varepsilon_{1}^{4}
\end{aligned}
$$

Here the Young's inequality is employed with the parameters $p=\frac{4}{3}, q=1, \alpha=1$, and applying to the term $g_{1} S_{1}^{3}\left(S_{2}+y_{2}\right)$ in Eq. (52), we obtain:

$g_{1} S_{1}^{3}\left(S_{2}+y_{2}\right) \leq \frac{3}{2} g_{1} S_{1}^{4}+\frac{1}{4} g_{1} S_{2}^{4}+\frac{1}{4} g_{1} y_{2}^{4}$

Now combining Eq. (53) and Eq. (52) and using the expression $b_{m} \leq g_{i} \leq b_{M}, 1 \leq i \leq n$ in (6), yields:

$$
\begin{aligned}
\mathrm{LV}_{1} \leq & -\underbrace{\left(k_{1}-\frac{3}{2}\right) b_{m}}_{c_{1}} S_{1}^{4}+\frac{b_{m}}{\lambda} \tilde{\theta}\left(\frac{\lambda}{2 a_{1}^{2}} S_{1}^{6} \zeta_{1}^{T}\left(Z_{1}\right) \zeta_{1}\left(Z_{1}\right)-\dot{\hat{\theta}}\right)+\frac{1}{4} g_{1} S_{2}^{4}+\frac{1}{4} b_{M} y_{2}^{4}+\frac{1}{2} a_{1}^{2} b_{M}^{2}+\frac{1}{4} \varepsilon_{1}^{4} \\
& \leq \underbrace{-c_{1} S_{1}^{4}+\frac{b_{m}}{\lambda} \tilde{\theta}\left(\frac{\lambda}{2 a_{1}^{2}} S_{1}^{6} \zeta_{1}^{T}\left(Z_{1}\right) \zeta_{1}\left(Z_{1}\right)-\dot{\hat{\theta}}\right)+\frac{1}{4} b_{M} y_{2}^{4}+\frac{1}{2} a_{1}^{2} b_{M}^{2}+\frac{1}{4} \varepsilon_{1}^{4}}_{\overline{L V}_{1}}+1 / 4 g_{1} S_{2}^{4}, c_{i}=\left(k_{i}-3 / 2\right) b_{m}, 1 \leq i \leq n,
\end{aligned}
$$

$$
\overline{\mathrm{x}}_{2}=-k_{1} S_{1}-\frac{1}{2 a_{1}^{2}} S_{1}^{3} \widehat{\theta}_{1}^{T}\left(Z_{1}\right) \zeta_{1}\left(Z_{1}\right)
$$

and by integrating it with the $x_{2}$ term of Eq. (46) and simple mathematical manipulation, we have:

$$
\begin{aligned}
& L V_{1} \leq-k_{1} g_{1} S_{1}^{4}+g_{1} S_{1}^{3}\left(x_{2}-\frac{-}{\mathrm{x}_{2}}\right) \\
& \quad+\frac{b_{m}}{\lambda} \tilde{\theta}\left(\frac{\lambda}{2 a_{1}^{2}} S_{1}^{6} \zeta_{1}^{T}\left(Z_{1}\right) \zeta_{1}\left(Z_{1}\right)-\dot{\hat{\theta}}\right)+\frac{1}{2} a_{1}^{2} b_{M}^{2}+\frac{1}{4} \varepsilon_{1}^{4}
\end{aligned}
$$

Introducing a new state variable $z_{2}$, and let $\overline{\mathrm{x}}_{2}$ pass through a first-order filter with a time constant $\epsilon_{2}$ to obtain $z_{2}$ as:

$\epsilon_{2} \dot{z}_{2}+z_{2}=-\bar{x}_{2}$

define the second error surface as follows:

$S_{2} \triangleq x_{2}-z_{2}$

define the first filter error as follows:

$y_{2} \triangleq z_{2}-\frac{-}{\mathrm{x}_{2}}=-\epsilon_{2} \dot{z_{2}}$
Defining $\overline{\mathrm{L}} V 1_{1}$ as in the following yields Eq. (55):

$$
\begin{aligned}
\overline{L V}_{1} \leq & -c_{1} S_{1}^{4}+\frac{b_{m}}{\lambda} \tilde{\theta}\left(\frac{\lambda}{2 a_{1}^{2}} S_{1}^{6} \zeta_{1}^{T}\left(Z_{1}\right) \zeta_{1}\left(Z_{1}\right)-\dot{\hat{\theta}}\right) \\
& +\frac{1}{4} b_{M} y_{2}^{4}+\frac{1}{2} a_{1}^{2} b_{M}^{2}+\frac{1}{4} \varepsilon_{1}^{4} \\
L V_{1} \leq & \overline{L V}_{1}+\frac{1}{4} g_{1} S_{2}^{4}
\end{aligned}
$$$$
\text { Step } 2 i,(2 \leq i \leq n)
$$

In order to maintain a systematic analysis and design procedure, and also for the brevity of the paper, a new state variable is defined as $x_{(n+1)} \triangleq u$. Now the design procedure is pursued as previous design steps. The derivative of the second error surface in Eq. (50), or equivalently of its generalization, the $i$ th error surface in Eq. (65), is obtained as:

$$
d S_{i}=\left(g_{i} x_{(i+1)}+f_{i}+\frac{1}{\epsilon_{i}}\left(z_{i}-\frac{-}{\mathrm{x}_{i}}\right)\right) d t+\psi_{i} d \psi, 2 \leq i \leq n
$$

Defining a stochastic Lyapanov function as: 
$V_{i}=V_{(i-1)}+\frac{1}{4} S_{i}^{4}+\frac{1}{4} y_{i}^{4}$

And applying the Ito's lemma to Eq. (57) results in:

$$
\begin{aligned}
\mathrm{LV}_{i}= & \overline{\mathrm{LV}}_{(i-1)}+\frac{1}{4} g_{(i-1)} S_{i}^{4} \\
& +S_{i}^{3}\left(g_{i} x_{(i+1)}+f_{i}+\frac{1}{\epsilon_{i}}\left(z_{i}-\bar{x}_{i}\right)\right) \\
& +\underbrace{\frac{3}{2} S_{i}^{2} \psi_{i}^{T} \psi_{i}}_{*}+y_{i}^{3}\left(-\frac{y_{i}}{\epsilon_{i}}+B_{i}\right)+\frac{3}{2} y_{i} \operatorname{Tr}\left\{G_{i}^{T} G_{i}\right\}
\end{aligned}
$$

where $B_{i}(\bullet)$, and $\operatorname{Tr}\left\{G_{i}^{T}(\bullet) G_{i}(\bullet)\right\}$ are continuous and smooth functions, which have maximums of $M_{i}$, and $N_{i}$ respectively. By applying the same method in Eq. (39) to the '*' term of Eq. (58), and using Young's inequality with the parameters of $i=2$ as $X=r_{2}, r_{2} \geq 0, Y=\frac{S_{2}^{2} S_{1}^{2} \varphi_{21}^{2}}{r_{2}}$, $(p, q, \alpha)=(2,2, \sqrt{2})$, similar generalization ${ }^{3}$ is driven for $2 \leq i \leq n$ :

$$
\begin{aligned}
& L V_{i} \leq_{\operatorname{LV}_{(i-1)}}^{-}+S_{i}^{3}\left(g_{i} x_{(i+1)}+f_{i}+\frac{1}{\epsilon_{i}}\left(z_{i}-\frac{-}{\mathrm{x}_{i}}\right)\right) \\
& \quad+\frac{1}{4} g_{(i-1)} S_{i}^{4}+\frac{3}{2} i S_{i}^{4} \varphi_{i i}^{2}+\frac{3}{4} r_{i}^{2}+\frac{3}{4} r_{i}^{-2} i^{2} S_{i}^{4}\left(\sum_{j=1}^{i-1} S_{j}^{2} \varphi_{i j}^{2}\right)^{2} \\
& \quad+y_{i}^{3}\left(-\frac{y_{i}}{\epsilon_{i}}+B_{i}\right)+\frac{3}{2} y_{i} \operatorname{Tr}\left\{G_{i}^{T} G_{i}\right\}
\end{aligned}
$$

An unknown function ${ }_{\mathrm{f}_{i}}$ is defined as follows [25]:

$$
\begin{aligned}
\overline{\mathrm{f}}_{i}= & f_{i}+\frac{1}{\epsilon_{i}}\left(z_{i}-\frac{-}{\mathrm{x}_{i}}\right)+\frac{1}{4} g_{(i-1)} S_{i}+\frac{3}{2} i S_{i} \varphi_{i i}^{2} \\
& +\frac{3}{4} r_{i}^{-2} i^{2} S_{i}\left(\sum_{j=1}^{i-1} S_{j}^{2} \varphi_{i j}^{2}\right)^{2}+\frac{3}{4} g_{i}^{\frac{4}{3}} S_{i}
\end{aligned}
$$

and substituting in Eq. (59) yields [12]:

$$
\begin{aligned}
\mathrm{LV}_{i} \leq & \overline{\mathrm{LV}}_{(i-1)}+S_{i}^{3}\left(g_{i} x_{(i+1)}+\bar{f}_{i}\right)-\frac{3}{4} g_{i}^{\frac{4}{3}} S_{i}^{4}+\frac{3}{4} r_{i}^{2}+y_{i}^{3}\left(-\frac{y_{i}}{\epsilon_{i}}+B_{i}\right) \\
& +\frac{3}{2} y_{i} \operatorname{Tr}\left\{G_{i}^{T} G_{i}\right\} \leq \overline{\mathrm{LV}}_{(i-1)}+g_{i} S_{i}^{3}(x_{(i+1)}+\underbrace{g_{i}^{-1} \bar{f}_{i}}_{\text {unknownterm }}) \\
& -\frac{3}{4} g_{i}^{\frac{4}{3}} S_{i}^{4}+\frac{3}{4} r_{i}^{2}+y_{i}^{3}\left(-\frac{y_{i}}{\epsilon_{i}}+B_{i}\right)+\frac{3}{2} y_{i} \operatorname{Tr}\left\{G_{i}^{T} G_{i}\right\}
\end{aligned}
$$

${ }^{3}$ A generalization of the technique employed in Eq. (B.5) will result in:

$$
\frac{3}{2} S_{i}^{2} \psi_{i}^{T} \psi_{i} \leq \frac{3}{2} i S_{i}^{4} \varphi_{i i}^{2}+\frac{3}{4} r_{i}^{2}+\frac{3}{4} r_{i}^{-2} i^{2} S_{i}^{4}\left(\sum_{j=1}^{i-1} S_{j}^{2} \varphi_{i j}^{2}\right)^{2}
$$

Similar to the previous design step, the specified unknown term is approximated using an RBFNN (Eq. 43), and relying on Eq. (31) we have:

$$
\begin{aligned}
& g_{i} S_{i}^{3} h_{i}\left(Z_{i}\right) \leq \frac{b_{m}}{2 a_{i}^{2}} S_{i}^{6} \theta \zeta_{i}^{T}\left(Z_{i}\right) \zeta_{i}\left(Z_{i}\right)+\frac{1}{2} a_{i}^{2} b_{M}^{2}+\frac{3}{4} g_{i}^{\frac{4}{3}} S_{i}^{4}+\frac{1}{4} \varepsilon_{i}^{4} \\
& \mathrm{LV}_{i} \leq \overline{\mathrm{LV}}_{(i-1)}+g_{i} S_{i}^{3} x_{(i+1)}+\frac{b_{m}}{2 a_{i}^{2}} S_{i}^{6} \theta \zeta_{i}^{T}\left(Z_{i}\right) \zeta_{i}\left(Z_{i}\right) \\
& +\frac{1}{2} a_{i}^{2} b_{M}^{2}+\frac{1}{4} \varepsilon_{i}^{4}+\frac{3}{4} r_{i}^{2}+y_{i}^{3}\left(-\frac{y_{i}}{\int_{i}}+B_{i}\right)+\frac{3}{2} y_{i} \operatorname{Tr}\left\{G_{i}^{T} G_{i}\right\}
\end{aligned}
$$

Simple mathematical manipulation based on using $\overline{\mathrm{X}}_{(i+1)}$ as the control input, $u$, yields:

$$
\begin{aligned}
& \overline{\mathrm{x}}_{(i+1)}=-k_{i} S_{i}-\frac{1}{2 a_{i}^{2}} S_{i}^{3} \widehat{\theta}_{i}^{T}\left(Z_{i}\right) \zeta_{i}\left(Z_{i}\right), 2 \leq i \leq n-1 \\
& L V_{i} \leq{ }_{\mathrm{L} V(i-1)}^{-}+g_{i} S_{i}^{3}\left(x_{(i+1)}-\overline{\mathrm{x}}_{(i+1)}\right)-k_{i} g_{i} S_{i}^{4} \\
& +\frac{b_{m}}{2 a_{i}^{2}} S_{i}^{6} \sim \zeta_{i}^{T}\left(Z_{i}\right) \zeta_{i}\left(Z_{i}\right)+\frac{1}{2} a_{i}^{2} b_{M}^{2}+\frac{1}{4} \varepsilon_{i}^{4}+\frac{3}{4} r_{i}^{2} \\
& \quad+y_{i}^{3}\left(-\frac{y_{i}}{\epsilon_{i}}+B_{i}\right)+\frac{3}{2} y_{i} \operatorname{Tr}\left\{G_{i}^{T} G_{i}\right\}, 2 \leq i \leq n-1
\end{aligned}
$$

Now, the control input $\overline{\mathrm{X}}_{(i+1)}$ is low-pass-filtered by a first-order filter $\dot{z}_{(i+1)}$ :

$\epsilon_{(i+1)} \dot{z}_{(i+1)}+z_{i+1}=\overline{\mathrm{x}}_{(i+1)}, 2 \leq i \leq n-1$

The error surface defined by $S$, along with the filter error $y$, is found at each step as follows:

$$
\begin{array}{r}
S_{(i+1)} \triangleq x_{(i+1)}-z_{(i+1)}, 2 \leq i \leq n-1 \\
y_{(i+1)} \triangleq z_{(i+1)}-\bar{x}_{(i+1)}=-\epsilon_{(i+1)} \dot{z}_{(i+1)}, 2 \leq i \leq n-1
\end{array}
$$

The derivative of Lyapunov function is consequently obtained as follows

$$
\begin{aligned}
L V_{i} \leq & \overline{L V}_{(i-1)}+g_{i} S_{i}^{3}\left(S_{(i+1)}+y_{(i+1)}+\bar{x}_{(i+1)}-\bar{x}_{(i+1)}\right)-k_{i} g_{i} S_{i}^{4} \\
& +\frac{b_{m}}{2 a_{i}^{2}} S_{i}^{6} \tilde{\theta} \zeta_{i}^{T}\left(Z_{i}\right) \zeta_{i}\left(Z_{i}\right)+\frac{1}{2} a_{i}^{2} b_{M}^{2}+\frac{1}{4} \varepsilon_{i}^{4}+\frac{3}{4} r_{i}^{2} \\
& +y_{i}^{3}\left(-\frac{y_{i}}{\epsilon_{i}}+B_{i}\right)+\frac{3}{2} y_{i} \operatorname{Tr}\left\{G_{i}^{T} G_{i}\right\} \leq \overline{L V}_{(i-1)} \\
& +\underbrace{g_{i} S_{i}^{3}\left(S_{(i+1)}+y_{(i+1)}\right)}_{*}-k_{i} g_{i} S_{i}^{4} \\
& +\frac{b_{m}}{2 a_{i}^{2}} S_{i}^{6} \tilde{\theta} \zeta_{i}^{T}\left(Z_{i}\right) \zeta_{i}\left(Z_{i}\right)+\frac{1}{2} a_{i}^{2} b_{M}^{2}+\frac{1}{4} \varepsilon_{i}^{4} \\
& +\frac{3}{4} r_{i}^{2}+y_{i}^{3}\left(-\frac{y_{i}}{\int_{i}}+B_{i}\right)+\frac{3}{2} y_{i} \operatorname{Tr}\left\{G_{i}^{T} G_{i}\right\}, \quad 2 \leq i \leq n-1
\end{aligned}
$$

Considering the term ' $*$ ' can be written as: 


$$
\begin{aligned}
g_{i} S_{i}^{3}\left(S_{(i+1)}+y_{(i+1)}\right) & \leq \frac{3}{2} g_{i} S_{i}^{4}+\frac{1}{4} g_{i} S_{(i+1)}^{4} \\
+ & \frac{1}{4} g_{i} y_{(i+1)}^{4}, 2 \leq i \leq n-1
\end{aligned}
$$

and using Eq. (54) for $i=2$, and also taking Eq. (1) into account in which $b_{m} \leq g_{i} \leq b_{M}, 1 \leq i \leq n$, the derivative of the Lyapunov function becomes:

$$
\begin{aligned}
L V_{i} & \leq\left(-\sum_{j=1}^{(i-1)} c_{j} S_{j}^{4}+\frac{b_{m}}{\lambda} \tilde{\theta}\left(\sum_{j=1}^{(i-1)} \frac{\lambda}{2 a_{j}^{2}} S_{j}^{6} \zeta_{j}^{T}\left(Z_{j}\right) \zeta_{j}\left(Z_{j}\right) \dot{\hat{\theta}}\right)\right. \\
& +\frac{1}{2} \sum_{j=1}^{(i-1)} a_{j}^{2} b_{M}^{2}+\frac{1}{4} \sum_{j=1}^{(i-1)} \varepsilon_{j}^{4} \\
& +\sum_{j=2}^{(i-1)}\left(\frac{b_{M}}{4}+\frac{3}{4}\left(\xi_{j} M_{j}\right)^{\frac{4}{3}}+\frac{3}{4}\left(\vartheta_{j} N_{j}\right)^{2}-\frac{1}{\int_{j}}\right) y_{j}^{4} \\
& \left.+\sum_{j=2}^{(i-1)}\left(\frac{1}{4 \xi_{j}^{4}}+\frac{3}{4 \vartheta_{j}^{2}}\right)\right)-(\underbrace{k_{i}-\frac{3}{2} b_{m}}_{c_{i}}) S_{i}^{4}+\frac{1}{4} b_{M} S_{(i+1)}^{4} \\
& +\frac{1}{4} b_{M} y_{(i+1)}^{4}+\underbrace{\frac{b_{m}}{2 a_{i}^{2}} S_{i}^{6} \tilde{\theta} \zeta_{i}^{T}\left(Z_{i}\right) \zeta_{i}\left(Z_{i}\right)}_{*}+\frac{1}{2} a_{i}^{2} b_{M}^{2}+\frac{1}{4} \varepsilon_{i}^{4} \\
& +\frac{3}{4} r_{i}^{2}-\frac{y_{i}^{4}}{\int_{i}}+\underbrace{}_{y_{i}^{3} B_{i}+\frac{3}{2} y_{i} T r\left\{G_{i}^{T} G_{i}\right\}}
\end{aligned}
$$

where $c_{i}=\left(k_{i}-\frac{3}{2}\right) b_{m}$. Applying the Young's inequality with the parameter set of $(p, q, \alpha)=\left(4 / 3,4, \xi_{i} M_{i}\right)$ and $(p, q, \alpha)=\left(2,2, \vartheta_{\mathrm{i}} N_{i}\right)$ to the first and the second term of '*' in Eq. (68), respectively, results in:

$$
\begin{aligned}
& y_{i}^{3} B_{i} \leq y_{i}^{3} M_{i} \leq \frac{3}{4}\left(\xi_{i} M_{i}\right)^{\frac{4}{3}} y_{i}^{4}+\frac{1}{4\left(\xi_{i} M_{i}\right)^{4}} M_{i}^{4} \\
& \frac{3}{2} y_{i}^{2} \operatorname{Tr}\left\{G_{i}^{T} G_{i}\right\} \leq \frac{3}{2} y_{i}^{2} N_{i} \leq \frac{3}{4}\left(\vartheta_{i} N_{i}\right)^{2} y_{i}^{4}+\frac{3}{4\left(\vartheta_{i} N_{i}\right)^{2}} N_{i}^{2} \\
& y_{i}^{3} B_{i}+\frac{3}{2} y_{i} \operatorname{Tr}\left\{G_{i}^{T} G_{i}\right\} \leq \frac{3}{4}\left(\xi_{i} M_{i}\right)^{\frac{4}{3}}+\frac{3}{4}\left(\vartheta_{i} N_{i}\right)^{2}+\frac{1}{4 \xi_{i}^{4}}+\frac{3}{4 \vartheta_{i}^{2}}
\end{aligned}
$$

where $\xi_{i}$ and $\vartheta_{i}$ are constants. Therefore, Eq. (68) becomes:

$$
\begin{aligned}
\mathrm{LV}_{i} \leq & \left(-\sum_{j=1}^{i} c_{j} S_{j}^{4}+\frac{b_{m}}{\lambda} \tilde{\theta}\left(\sum_{j=1}^{i} \frac{\lambda}{2 a_{j}^{S}} S_{j}^{6} \zeta_{j}^{T}\left(Z_{j}\right) \zeta_{j}\left(Z_{j}\right)-\dot{\hat{\theta}}\right)\right. \\
& +\frac{1}{2} \sum_{j=1}^{i} a_{j}^{2} b_{M}^{2}+\frac{1}{4} \sum_{j=1}^{i} \varepsilon_{j}^{4}+\sum_{j=2}^{i}\left(\frac{b_{M}}{4}+\frac{3}{4}\left(\xi_{j} M_{j}\right)^{\frac{4}{3}}+\frac{3}{4}\left(\vartheta_{j} N_{j}\right)^{2}-\frac{1}{\int_{j}}\right) y_{j}^{4} \\
& \left.+\sum_{j=2}^{i}\left(\frac{1}{4 \xi_{j}^{4}}+\frac{3}{4 \vartheta_{j}^{2}}\right)+\frac{3}{4} \sum_{j=2}^{i} r_{j}^{2}\right)+\frac{1}{4} b_{M} y_{(i+1)}^{4} \\
+ & \frac{1}{4} b_{M} S_{(i+1)}^{4}, 3 \leq i \leq n-1
\end{aligned}
$$

The above inequality is simplified by defining $\overline{\mathrm{L}}_{V_{i}}$ as follows:

$$
\begin{aligned}
L V_{i} \leq & \overline{L V_{i}}+\frac{1}{4} g_{i} S_{(i+1)}^{4}, 3 \leq i \leq n-1 \\
-\overline{\mathrm{L} V_{i}}= & -\sum_{j=1}^{i} c_{j} S_{j}^{4}+\frac{b_{m}}{\lambda} \tilde{\theta}\left(\sum_{j=1}^{i} \frac{\lambda}{2 a_{j}^{2}} S_{j}^{6} \zeta_{j}^{T}\left(Z_{j}\right) \zeta_{j}\left(Z_{j}\right)-\dot{\hat{\theta}}\right) \\
& +\frac{1}{2} \sum_{j=1}^{i} a_{j}^{2} b_{M}^{2}+\frac{1}{4} \sum_{j=1}^{i} \varepsilon_{j}^{4} \\
& +\sum_{j=2}^{i}\left(\frac{b_{M}}{4}+\frac{3}{4}\left(\xi_{j} M_{j}\right)^{\frac{4}{3}}+\frac{3}{4}\left(\vartheta_{j} N_{j}\right)^{2}-\frac{1}{\epsilon_{j}}\right) y_{j}^{4} \\
& +\sum_{j=2}^{i}\left(\frac{1}{4 \xi_{j}^{4}}+\frac{3}{4 \vartheta_{j}^{2}}\right)+\frac{3}{4} \sum_{j=2}^{i} r_{j}^{2}+\frac{1}{4} b_{M} y_{(i+1)}^{4}
\end{aligned}
$$

Then, the Itô's formula for the stochastic Lyapunov function of the $n$th step is derived as follows:

$$
\begin{aligned}
& L V_{n} \leq-\sum_{j=1}^{n} c_{j} S_{j}^{4}+\frac{b_{m}}{\lambda} \tilde{\theta}\left(\sum_{j=1}^{n} \frac{\lambda}{2 a_{j}^{2}} S_{j}^{6} \zeta_{j}^{T}\left(Z_{j}\right) \zeta_{j}\left(Z_{j}\right)-\dot{\hat{\theta}}\right) \\
& +\frac{1}{2} \sum_{j=1}^{n} a_{j}^{2} b_{M}^{2}+\frac{1}{4} \sum_{j=1}^{n} \varepsilon_{j}^{4}+\sum_{j=2}^{n}\left(\frac{b_{M}}{4}+\frac{3}{4}\left(\xi_{j} M_{j}\right)^{\frac{4}{3}}+\frac{3}{4}\left(\vartheta_{j} N_{j}\right)^{2}-\frac{1}{\epsilon_{j}}\right) y_{j}^{4} \\
& \quad+\sum_{j=2}^{n}\left(\frac{1}{4 \xi_{j}^{4}}+\frac{3}{4 \vartheta_{j}^{2}}\right)+\frac{3}{4} \sum_{j=2}^{n} r_{j}^{2} .
\end{aligned}
$$

The above equation is simplified by using the following expressions:

$$
\begin{aligned}
& L V_{n} \leq-\sum_{j=1}^{n} c_{j} S_{j}^{4}-\sum_{j=2}^{n} d_{j} y_{j}^{4}+\frac{k_{0} b_{m}}{\lambda} \underbrace{\tilde{\theta} \hat{\theta}}_{*}+\beta_{0}, \\
& \dot{\hat{\theta}}=\sum_{j=1}^{n} \frac{\lambda}{2 a_{j}^{2}} S_{j}^{6} \zeta_{j}^{T}\left(V Z_{j}\right) \zeta_{j}\left(Z_{j}\right)-k_{0} \widehat{\theta} \\
& \begin{array}{l}
\frac{1}{2} \sum_{j=1}^{n} a_{j}^{2} b_{M}^{2}+\frac{1}{4} \sum_{j=1}^{n} \varepsilon_{j}^{4}+\sum_{j=2}^{n}\left(\frac{1}{4 \xi_{j}^{4}}+\frac{3}{4 \vartheta_{j}^{2}}\right)+\frac{3}{4} \sum_{j=2}^{n} r_{j}^{2} \\
\quad=\beta_{0}>0
\end{array}
\end{aligned}
$$

$$
\left(\frac{1}{\epsilon_{j}}-\frac{b_{M}}{4}-\frac{3}{4}\left(\xi_{j} M_{j}\right)^{\frac{4}{3}}-\frac{3}{4}\left(\vartheta_{j} N_{j}\right)^{2}\right)=d_{j}>0,2 \leq j \leq n
$$

The term '*' can be modified using the Young's inequality as follows:

$$
\begin{aligned}
& \tilde{\theta}=\sim(\theta-\tilde{\theta})=-\sim_{\theta}^{2}+\underset{\theta}{\theta} \\
& \widetilde{\theta}=-\sim_{\theta}^{2}+\underset{\theta}{\theta} \leq-\sim^{2}+\left(\frac{\tilde{\theta}^{2}}{2}+\frac{\theta^{2}}{2}\right) \leq-\frac{\tilde{\theta}^{2}}{2}+\frac{\theta^{2}}{2}
\end{aligned}
$$

Hence, Eq. (72) becomes: 


$$
\begin{gathered}
L V_{n} \leq-\sum_{j=1}^{n} c_{j} S_{j}^{4}-\sum_{j=2}^{n} d_{j} y_{j}^{4}+\frac{k_{0} b_{m}}{\lambda}\left(-\frac{\tilde{\theta}^{2}}{2}+\frac{\theta^{2}}{2}\right)+\beta_{0} \leq \\
-\sum_{j=1}^{n} c_{j} S_{j}^{4}-\sum_{j=2}^{n} d_{j} y_{j}^{4}-\frac{k_{0} b_{m}}{2 \lambda} \tilde{\theta}^{2}+\underbrace{\frac{k_{0} b_{m}}{2 \lambda} \theta^{2}+\beta_{0}}_{\beta_{1}}
\end{gathered}
$$

$\mathrm{LV}_{n} \leq-\sum_{j=1}^{n} c_{j} S_{j}^{4}-\sum_{j=2}^{n} d_{j} y_{j}^{4}-\frac{k_{0} b_{m}}{2 \lambda} \tilde{\theta}^{2}+\beta_{1} \leq-\alpha_{1} V+\beta_{1}$, $\alpha_{1}=\min \left\{4 c_{j}, 4 d_{j}, k_{0}, j=1,2, \ldots, n\right\}, \beta_{1}=\beta_{0}+\frac{k_{0} b_{m}}{2 \lambda} \theta^{2}$

where $\quad \alpha_{1}=\min \left\{4 c_{j}, 4 d_{j}, k_{0}, j=1,2, \ldots, n\right\}, \quad$ and $\beta_{1}=\beta_{0}+\frac{k_{0} b_{m}}{2 \lambda} \theta^{2}$. Consequently, the nth stochastic Lyapunov function is:

$L V_{n} \leq-\alpha_{1} V+\beta_{1}$

According to Lemma 1 of Appendix 1 using the control signal, $u$, all the closed-loop signals of the entire system remain bounded in probability, and the output signal, $y=x_{1}$, tracks the reference input of the system.

\section{Appendix 4}

\section{Proof of theorem 2 (effect of hysteresis in actuators):}

Assuming the system, described in (1), is subjected to an actuator hysteresis defined by a Prandtl-Ishlinskii (PI) model. The play operator of the model is defined by Eqs. (2)-(6). The control system and the input to the model are defined by Eq. (12) and Eq. (5), respectively. If the analysis and design procedure is pursued as in Section B, all the steps would be similar to Eqs. (35)- (67). The derivative of the error surface is found relying on Eq. (56) using the last term of Eq. (35):

$$
\begin{aligned}
d S_{n}= & \left(g_{n} p_{0} v(t)-g_{n} d[v](t)+f_{n}+\frac{1}{\epsilon_{n}}\left(z_{n}-\frac{-}{\mathrm{x}_{n}}\right)\right) d t \\
& +\psi_{n} d \psi
\end{aligned}
$$

In order to investigate stability of the system, a stochastic Lyapunov function is chosen as:

$V_{n}=V_{n-1}+\frac{1}{4} S_{n}^{4}+\frac{1}{4} y_{n}^{4}$

where $y_{i+1}, 2 \leq i \leq n-1$ is the error of the $i$ th filters, which are defined by Eq. (51) and Eq. (65). Using the Itô's formula along with Eq. (36) in Eq. (37) yields:

$$
\begin{aligned}
L V_{n}= & L V_{(n-1)} \\
& +S_{n}^{3}\left(g_{n} p_{0} v(t)-g_{n} d[v](t)+f_{n}+\frac{1}{\epsilon_{n}}\left(z_{n}-\frac{-}{\mathrm{x}_{n}}\right)\right) \\
& +\frac{3}{2} S_{n}^{2} \psi_{n}^{T} \psi_{n}+y_{n}^{3}\left(-\frac{y_{n}}{\epsilon_{n}}+B_{n}\right)+\frac{3}{2} y_{n} \operatorname{Tr}\left\{G_{n}^{T} G_{n}\right\}
\end{aligned}
$$

where $B_{i}(\bullet)$ and $\operatorname{Tr}\left\{G_{i}^{T}(\bullet) G_{i}(\bullet)\right\}$ are continuous and smooth functions, showing maximums of $M_{i}$, and $N_{i}$ respectively. By following similar design procedure as described in the previous sequel by derivations Eq. (58)Eq. (68), it can be easily found that the above inequality becomes:

$$
\begin{aligned}
L V_{n} & \leq \underset{\operatorname{LV} V_{(n-1)}}{-}+\frac{1}{4} g_{(n-1)} S_{n}^{4} \\
& +S_{n}^{3}\left(g_{n} p_{0} v(t)-g_{n} d[v](t)+f_{n}+\frac{1}{\epsilon_{n}}\left(z_{n}-\frac{-}{\mathrm{x}_{n}}\right)\right) \\
& +\frac{3}{2} n S_{n}^{4} \varphi_{n n}^{2}+\frac{3}{4} r_{n}^{2}+y_{n}^{3}\left(-\frac{y_{n}}{\epsilon_{n}}+B_{n}\right) \\
& +\frac{3}{4} r_{n}^{-2} n^{2} S_{n}^{4}\left(\sum_{j=1}^{n-1} S_{j}^{2} \varphi_{i j}^{2}\right)^{2}+\frac{3}{2} y_{n} \operatorname{Tr}\left\{G_{n}^{T} G_{n}\right\}
\end{aligned}
$$

By defining $\overline{\mathrm{f}}_{n}$ as follows:

$$
\begin{aligned}
\overline{\mathrm{f}}_{n}= & f_{n}+\frac{1}{\epsilon_{n}}\left(z_{n}-\frac{-}{\mathrm{x}_{n}}\right)+\frac{3}{2} n S_{n} \varphi_{n n}^{2}+\frac{1}{4} g_{(n-1)} S_{n} \\
& +\frac{3}{4} r_{n}^{-2} n^{2} S_{n}\left(\sum_{j=1}^{n-1} S_{j}^{2} \varphi_{i j}^{2}\right)^{2}+\frac{3}{4} g_{n}^{\frac{4}{3}} S_{n}-g_{n} d[v](t)
\end{aligned}
$$

Eq. (80) becomes:

$$
\begin{aligned}
\mathrm{LV}_{n} \leq & \overline{\mathrm{LV}_{(n-1)}}+S_{n}^{3}(\underbrace{g_{n} p_{0}}_{g_{n}} v(t)+\bar{f}_{n})-\frac{3}{4}{\overline{g_{n}}}^{\frac{4}{3}} S_{n} \\
& +\frac{3}{4} r_{n}^{2}+y_{n}^{3}\left(-\frac{y_{n}}{\epsilon_{n}}+B_{n}\right)+\frac{3}{2} y_{n} \operatorname{Tr}\left\{G_{n}^{T} G_{n}\right\} \leq \overline{\mathrm{LV}}_{(n-1)} \\
& +\overline{g_{n}} S_{n}^{3}(v(t)+\underbrace{\bar{g}^{-1} \bar{f}_{n}}_{\text {unknownterm }})-\frac{3}{4} \bar{g}_{n^{3}}^{\frac{4}{3}} S_{n}+\frac{3}{4} r_{n}^{2} \\
& +y_{n}^{3}\left(-\frac{y_{n}}{\epsilon_{n}}+B_{n}\right)+\frac{3}{2} y_{n} \operatorname{Tr}\left\{G_{n}^{T} G_{n}\right\}
\end{aligned}
$$

where $\overline{\mathrm{g}}_{n}=g_{n} p_{0}$. The unknown term in Eq. (107) is approximated using an RBFNN as follows:

$$
\begin{aligned}
h_{n}\left(Z_{n}\right) & =\underset{\mathrm{g}_{n}}{{ }^{-1} \mathrm{f}_{n}}=W_{n}^{* T} \zeta_{n}\left(Z_{n}\right)+\delta_{n}^{*}\left(Z_{n}\right),\left|\delta_{n}\left(Z_{n}\right)\right| \leq \varepsilon_{n}, Z_{n} \\
& =[x, \theta]
\end{aligned}
$$

The derivative of the Lyapunov function leads to: 


$$
\begin{aligned}
& L V_{n} \leq \underset{\mathrm{L} V_{(n-1)}}{-}+\frac{-}{\mathrm{g}_{n}} S_{n}^{3}\left(v(t)+h_{n}\left(Z_{n}\right)\right)-\frac{3}{4} \mathrm{~g}_{n}^{-\frac{4}{3}} S_{n}+\frac{3}{4} r_{n}^{2} \\
& \quad+y_{n}^{3}\left(-\frac{y_{n}}{\epsilon_{n}}+B_{n}\right)+\frac{3}{2} y_{n} \operatorname{Tr}\left\{G_{n}^{T} G_{n}\right\} \leq \underset{\mathrm{L} V_{(n-1)}}{-}+b_{m} S_{n}^{3} v(t) \\
& \quad+\frac{-}{\mathrm{g}_{n}} S_{n}^{3} h_{n}\left(Z_{n}\right)-\frac{3}{4} \mathrm{~g}_{n}^{-\frac{4}{3}} S_{n}+\frac{3}{4} r_{n}^{2}+y_{n}^{3}\left(-\frac{y_{n}}{\epsilon_{n}}+B_{n}\right) \\
& \quad+\frac{3}{2} y_{n} \operatorname{Tr}\left\{G_{n}^{T} G_{n}\right\}
\end{aligned}
$$

Expanding the term $\overline{\mathrm{g}}_{n} S_{n}^{3} h_{n}\left(Z_{n}\right)$ in Eq. (84) using the derivation Eq. (34) and replacing in Eq. (3) yields:

$$
\begin{aligned}
& L V_{n} \leq \underset{\operatorname{LV_{(n-1)}}}{-}+b_{m} S_{n}^{3} v(t)+\frac{b_{m}}{2 a_{n}^{2}} S_{n}^{6} \theta \zeta_{n}^{T}\left(Z_{n}\right) \zeta_{n}\left(Z_{n}\right)+\frac{1}{2} a_{n}^{2} b_{M}^{2} \\
& \quad+\frac{1}{4} \varepsilon_{n}^{4}+\frac{3}{4} r_{n}^{2}+y_{n}^{3}\left(-\frac{y_{n}}{\epsilon_{n}}+B_{n}\right)+\frac{3}{2} y_{n} \operatorname{Tr}\left\{G_{n}^{T} G_{n}\right\}
\end{aligned}
$$

By choosing $v(t)=-k_{n} S_{n}-\frac{1}{2 a_{n}^{2}} S_{n}^{3} \widehat{\theta} \zeta_{n}^{T}\left(Z_{n}\right) \zeta\left(Z_{n}\right)$ and using Eq. (70), the above derivation becomes:

$$
\begin{aligned}
\mathrm{LV}_{n} \leq & \left(-\sum_{j=1}^{(n-1)} c_{j} S_{j}^{4}+\frac{b_{m}}{\lambda} \tilde{\theta}\left(\sum_{j=1}^{(n-1)} \frac{\lambda}{2 a_{j}^{2}} S_{j}^{6} \zeta_{j}^{T}\left(Z_{j}\right) \zeta_{j}\left(Z_{j}\right)-\dot{\hat{\theta}}\right)\right. \\
& +\frac{1}{2} \sum_{j=1}^{(n-1)} a_{j}^{2} b_{M}^{2}+\frac{1}{4} \sum_{j=1}^{(n-1)} \varepsilon_{j}^{4} \\
& +\sum_{j=2}^{(n-1)}\left(\frac{b_{M}}{4}+\frac{3}{4}\left(\xi_{j} M_{j}\right)^{\frac{4}{3}}+\frac{3}{4}\left(\vartheta_{j} N_{j}\right)^{2}-\frac{1}{\epsilon_{j}}\right) y_{j}^{4} \\
& \left.+\sum_{j=2}^{(n-1)}\left(\frac{1}{4 \xi_{j}^{4}}+\frac{3}{4 \vartheta_{j}^{2}}\right)+\frac{3}{4} \sum_{j=2}^{(n-1)} r_{j}^{2}+\frac{1}{4} b_{M} y_{(i+1)}^{4}\right) \\
& -\underbrace{k_{n} b_{m}}_{c_{n}} S_{n}^{4}+\frac{b_{m}}{2 a_{n}^{2}} S_{n}^{6} \tilde{\theta} \zeta_{n}^{T}\left(Z_{n}\right) \zeta_{n}\left(Z_{n}\right)+\frac{1}{2} a_{n}^{2} b_{M}^{2} \\
& +\frac{1}{4} \varepsilon_{n}^{4}+\frac{3}{4} r_{n}^{2}+y_{n}^{3}\left(-\frac{y_{n}}{\epsilon_{n}}+B_{n}\right)+\frac{3}{2} y_{n} \operatorname{Tr}\left\{G_{n}^{T} G_{n}\right\}
\end{aligned}
$$

where $c_{i}=\left(k_{i}-\frac{3}{2}\right) b_{m}, k_{i}$ are design parameters, $\xi_{i}$ and $\vartheta_{i}$ are constants. Furthermore, due to the boundedness of (6), Eq. (50) is also bounded, relying on the affecting term of (6) in Eq. (50). As shown before, Eq. (50) is similar to Eq. (69) for $i=n$. Hence, from this step onwards, the stability analysis is similar to Eq. (70)-Eq. (76) and gives the same results. Consequently, according to Lemma 1 of Appendix 1 using the virtual control input signals (14), the designed control input signal, $v(t)$, the $(n-1)$ low-pass filters (16), and the adaptive law of RBFNN weights (17), $\widehat{\theta}(t)$, all of the closed-loop signals remain bounded in sense of the probability, and therefore the output of the system $y=x_{1}$ tracks the reference input signal, $y_{r}$, with an arbitrarily small error. Thus, the proposed controller design method guarantees the closed-loop stability of the entire system at the presence on unknown actuator hysteresis, and the system dynamics.

\section{Appendix 5}

\section{Proof of theorem 3 (effect of dead-zone in actuator)}

Assume that the system (1) is subjected to an actuator dead-zone as defined by Eqs. (7), (8). Similar to the designing steps described in (19-61), rewriting Eq. (55) based on (13) gives:

$$
\begin{aligned}
d S_{n}= & \left(g_{n}\left(K^{T}(t) \Phi(t) v+d(v)\right)+f_{n}+\frac{1}{\epsilon_{n}}\left(z_{n}-\frac{-}{\mathrm{x}_{n}}\right)\right) d t \\
& +\psi_{n} d \psi \\
= & \left(g_{n} K^{T}(t) \Phi(t) v+g_{n} d(v)+f_{n}+\frac{1}{\epsilon_{n}}\left(z_{n}-\frac{-}{\mathrm{x}_{n}}\right)\right) d t \\
& +\psi_{n} d \psi
\end{aligned}
$$

Choosing a stochastic Lyapunov function as:

$V_{n}=V_{n-1}+\frac{1}{4} S_{n}^{4}+\frac{1}{4} y_{n}^{4}$

and using the Itô's formula, we have:

$$
\begin{aligned}
L V_{n}= & L V_{(n-1)} \\
& +S_{n}^{3}\left(g_{n}\left(K^{T}(t) \Phi(t) v+d(v)\right)+f_{n}+\frac{1}{\epsilon_{n}}\left(z_{n}-\frac{-}{\mathrm{x}_{n}}\right)\right) \\
& +\frac{3}{2} S_{n}^{2} \psi_{n}^{T} \psi_{n}+y_{n}^{3}\left(-\frac{y_{n}}{\epsilon_{n}}+B_{n}\right)+\frac{3}{2} y_{n} \operatorname{Tr}\left\{G_{n}^{T} G_{n}\right\}
\end{aligned}
$$

Relying on the following considerations:

Youngs Inequality : $S_{n}^{3} g_{n} d(v) \leq{ }_{4}^{3} g_{n} S_{n}^{4}+{ }_{4}^{1} b_{\max } p^{*}$

$\frac{3}{2} S_{n}^{2} \psi_{n}^{T} \psi_{n} \leq \frac{3}{2} n S_{n}^{4} \varphi_{n n}^{2}+\frac{3}{4} r_{n}^{2}+\frac{3}{4} r_{n}^{-2} n^{2} S_{n}^{4}\left(\sum_{j=1}^{n-1} S_{j}^{2} \varphi_{i j}^{2}\right)^{2}$

defining the auxiliary function $\overline{\mathrm{f}}_{n}$ and performing simple mathematical manipulations yield: 


$$
\begin{aligned}
\overline{\mathrm{f}_{n}}=f_{n} & +\frac{1}{\epsilon_{n}}\left(z_{n}-\frac{-}{\mathrm{x}_{n}}\right)+\frac{3}{2} n S_{n} \varphi_{n n}^{2} \\
& +\frac{3}{4} r_{n}^{-2} n^{2} S_{n}\left(\sum_{j=1}^{n-1} S_{j}^{2} \varphi_{i j}^{2}\right)^{2}+\frac{1}{4} g_{(n-1)} S_{n}+\frac{3}{4} g_{n}^{\frac{4}{3}} S_{n} \\
& +\frac{3}{4} g_{n} S_{n} \\
\mathrm{LV}_{n} \leq & \overline{\mathrm{LV}}_{(n-1)}+S_{n}^{3}(\underbrace{g_{n} K^{T}(t) \Phi(t)}_{g_{n}} v(t)+\bar{f}_{n}) \\
& -\frac{3}{4} \overline{g_{n}{ }^{3}} S_{n}+\frac{3}{4} r_{n}^{2}+y_{n}^{3}\left(-\frac{y_{n}}{\epsilon_{n}}+B_{n}\right) \\
& +\frac{3}{2} y_{n} \operatorname{Tr}\left\{G_{n}^{T} G_{n}\right\} \leq \overline{L V_{(n-1)}}+\overline{g_{n}} S_{n}^{3}(v(t)+\underbrace{\overline{g_{n}}-1 \bar{f}_{n}}_{\text {unknownterm }}) \\
& -\frac{3}{4} \overline{g_{n}{ }^{3}} S_{n}+\frac{3}{4} r_{n}^{2}+y_{n}^{3}\left(-\frac{y_{n}}{\epsilon_{n}}+B_{n}\right) \\
& +\frac{3}{2} y_{n} \operatorname{Tr}\left\{G_{n}^{T} G_{n}\right\}+\frac{1}{4} b_{\max } p^{*}
\end{aligned}
$$

where $\overline{\mathrm{g}}_{n}=g_{n} K^{T}(t) \Phi(t), \overline{\mathrm{L}}_{(n-1)}$ is defined in Eq. (55). Similar approximation is applied to the unknown term in Eq. (92) as in Eq. (61), using an RBFNN, which leads to:

$$
\begin{aligned}
\mathrm{LV}_{n} \leq & \overline{\mathrm{LV}}_{(n-1)}+\bar{g}_{n} S_{n}^{3}\left(v(t)+h_{n}\left(Z_{n}\right)\right)-\frac{3}{4} \bar{g}_{n}^{\frac{4}{3}} S_{n} \\
& +\frac{3}{4} r_{n}^{2}+y_{n}^{3}\left(-\frac{y_{n}}{\epsilon_{n}}+B_{n}\right)+\frac{3}{2} y_{n} \operatorname{Tr}\left\{G_{n}^{T} G_{n}\right\} \\
& +\frac{1}{4} b_{\max } \delta^{* 4} \leq \overline{L V_{(n-1)}}+b_{m} S_{n}^{3} v(t)+\overline{g_{n}} S_{n}^{3} h_{n}\left(Z_{n}\right) \\
& -\frac{3}{4} \bar{g}_{n^{3}} S_{n}+\frac{3}{4} r_{n}^{2}+y_{n}^{3}\left(-\frac{y_{n}}{\epsilon_{n}}+B_{n}\right) \\
& +\frac{3}{2} y_{n} \operatorname{Tr}\left\{G_{n}^{T} G_{n}\right\}+\frac{1}{4} b_{\max } p^{*}
\end{aligned}
$$

Using the same technique employed in Eq. (62) for the above equation yields:

$$
\begin{aligned}
& L V_{n} \leq \underset{\operatorname{LV}_{(n-1)}}{-}+b_{m} S_{n}^{3} v(t)+\frac{b_{m}}{2 a_{n}^{2}} S_{n}^{6} \theta \zeta_{n}^{T}\left(Z_{n}\right) \zeta_{n}\left(Z_{n}\right)+\frac{1}{2} a_{n}^{2} b_{M}^{2} \\
& \quad+\frac{1}{4} \varepsilon_{n}^{4}+\frac{3}{4} r_{n}^{2}+y_{n}^{3}\left(-\frac{y_{n}}{\epsilon_{n}}+B_{n}\right)+\frac{3}{2} y_{n} \operatorname{Tr}\left\{G_{n}^{T} G_{n}\right\} \\
& \quad+\frac{1}{4} b_{\max } p^{*}
\end{aligned}
$$

Choosing $v(t)$ as follows:

$v(t)=-k_{n} S_{n}-\frac{1}{2 a_{n}^{2}} S_{n}^{3} \widehat{\theta}^{2} \zeta_{n}^{T}\left(Z_{n}\right) \zeta\left(Z_{n}\right)$

Replacing Eq. (71) along with simple mathematical manipulations gives:

$$
\begin{aligned}
\mathrm{LV}_{n} \leq & \left(-\sum_{j=1}^{(n-1)} c_{j} S_{j}^{4}+\frac{b_{m}}{\lambda} \tilde{\theta}\left(\sum_{j=1}^{(n-1)} \frac{\lambda}{2 a_{j}^{2}} S_{j}^{6} \zeta_{j}^{T}\left(Z_{j}\right) \zeta_{j}\left(Z_{j}\right)-\dot{\hat{\theta}}\right)\right. \\
& +\frac{1}{2} \sum_{j=1}^{(n-1)} a_{j}^{2} b_{M}^{2}+\frac{1}{4} \sum_{j=1}^{(n-1)} \varepsilon_{j}^{4} \\
& +\sum_{j=2}^{(n-1)}\left(\frac{b_{M}}{4}+\frac{3}{4}\left(\xi_{j} M_{j}\right)^{\frac{4}{3}}+\frac{3}{4}\left(\vartheta_{j} N_{j}\right)^{2}-\frac{1}{\epsilon_{j}}\right) y_{j}^{4} \\
& \left.+\sum_{j=2}^{(n-1)}\left(\frac{1}{4 \xi_{j}^{4}}+\frac{3}{4 \vartheta_{j}^{2}}\right)+\frac{3}{4} \sum_{j=2}^{(n-1)} r_{j}^{2}+\frac{1}{4} b_{M} y_{(i+1)}^{4}\right) \\
& -c_{n} S_{n}^{4}+\frac{b_{m}}{2 a_{n}^{2}} S_{n}^{6} \tilde{\theta} \zeta_{n}^{T}\left(Z_{n}\right) \zeta_{n}\left(Z_{n}\right)+\frac{1}{2} a_{n}^{2} b_{M}^{2}+\frac{1}{4} \varepsilon_{n}^{4} \\
& +\frac{3}{4} r_{n}^{2}-\frac{y_{n}^{4}}{\epsilon_{n}}+\underbrace{y_{n}^{3} B_{n}+\frac{3}{2} y_{n} \operatorname{Tr}\left\{G_{n}^{T} G_{n}\right\}}_{*}+\frac{1}{4} b_{\max } p^{*}
\end{aligned}
$$

Using Eq. (68) for the '*' term in Eq. (96), and replacing in the above derivation yield:

$$
\begin{aligned}
L V_{n} & \leq-\sum_{j=1}^{n} c_{j} S_{j}^{4}+\frac{b_{m}}{\lambda} \tilde{\theta}\left(\sum_{j=1}^{n} \frac{\lambda}{2 a_{j}^{2}} S_{j}^{6} \zeta_{j}^{T}\left(Z_{j}\right) \zeta_{j}\left(Z_{j}\right)-\dot{\hat{\theta}}\right) \\
& +\frac{1}{2} \sum_{j=1}^{n} a_{j}^{2} b_{M}^{2}+\frac{1}{4} \sum_{j=1}^{n} \varepsilon_{j}^{4}+\sum_{j=2}^{n}\left(\frac{1}{4 \xi_{j}^{4}}+\frac{3}{4 \vartheta_{j}^{2}}\right) \\
& +\sum_{j=2}^{n}\left(\frac{b_{M}}{4}+\frac{3}{4}\left(\xi_{j} M_{j}\right)^{\frac{4}{3}}+\frac{3}{4}\left(\vartheta_{j} N_{j}\right)^{2}-\frac{1}{\epsilon_{j}}\right) y_{j}^{4}+\frac{3}{4} \sum_{j=2}^{n} r_{j}^{2} \\
& +\frac{1}{4} b_{\text {max }} p^{*}
\end{aligned}
$$

Now choosing the following expression results:

$$
\dot{\hat{\theta}}=\sum_{j=1}^{n} \frac{\lambda}{2 a_{j}^{2}} S_{j}^{6} \zeta_{j}^{T}\left(Z_{j}\right) \zeta_{j}\left(Z_{j}\right)-k_{0} \widehat{\theta}
$$

$\frac{1}{2} \sum_{j=1}^{n} a_{j}^{2} b_{M}^{2}+\frac{1}{4} \sum_{j=1}^{n} \varepsilon_{j}^{4}+\sum_{j=2}^{n}\left(\frac{1}{4 \xi_{j}^{4}}+\frac{3}{4 \vartheta_{j}^{2}}\right)+\frac{3}{4} \sum_{j=2}^{n} r_{j}^{2}$

$$
\begin{aligned}
& \quad+\frac{1}{4} b_{\max } p^{*} \\
& \quad=\beta_{0}>0 \\
& \left(\frac{1}{\epsilon_{j}}-\frac{b_{M}}{4}-\frac{3}{4}\left(\xi_{j} M_{j}\right)^{\frac{4}{3}}-\frac{3}{4}\left(\vartheta_{j} N_{j}\right)^{2}\right)=d_{j}>0,2 \leq j \leq n
\end{aligned}
$$

As it is shown, in comparison with Eq. (73), $\beta_{0}$ is the only changed term and from this step onwards this proof is similar to Eq. (74)-Eq. (76) proving the system stability. Based on Lemma 1 of Appendix 1 using the virtual control input signals (19), the designed control input signal, $v(t)$, the $(n-1)$ low-pass filters (21), and the adaptive law of RBFNN weights (22), $\widehat{\theta}(t)$, all of the closed-loop signals remain bounded in sense of the probability, and the output 
of the system $y=x_{1}$ tracks the reference input signal of the system, $y_{r}$, with an arbitrarily small error. Thus, the proposed controller design method guarantees the closed-loop stability of the entire system in the presence of unknown dead-zone in actuator, and system dynamics.

\section{Appendix 6}

\section{Proof of theorem 5 (direct ANNDSC compensation of Prandtl-Ishlinskii hysteresis in strict-feedback nonlinear stochastic systems)}

A direct adaptive neural network controller is designed specifically for nonlinear stochastic systems in strict-feedback form (6) subjected to an unknown Prandtl-Ishlinskii (PI) hysteresis as defined in Eqs. (2) to (6). Rewriting Eq. (56) for $n$th design step and simple mathematical manipulations yields:

$$
\begin{aligned}
d S_{n}= & \left(g_{n}\left(p_{0} v(t)-d[v](t)\right)+f_{n}+\frac{1}{\epsilon_{n}}\left(z_{n}-\frac{-}{\mathrm{x}_{n}}\right)\right) d t \\
& +\psi_{n} d \psi \\
= & \left(g_{n} p_{0} v(t)+f_{n}+\frac{1}{\epsilon_{n}}\left(z_{n}-\frac{-}{\mathrm{x}_{n}}\right)\right) d t \\
& -g_{n} p_{0}\left(\int_{0}^{R} p_{p_{0}}(t, r) F_{r}[v](t) d r\right) d t+\psi_{n} d \psi
\end{aligned}
$$

where $p_{p_{0}}(t, r)=p(t, r) / p_{0}$ [10]. Choosing a stochastic Lyapunov function as:

$V_{n}=V_{n-1}+\frac{1}{4} S_{n}^{4}+\frac{1}{4} y_{n}^{4}+\frac{b_{M}}{2 \gamma_{p r}} \int_{0}^{R}{ }_{0}^{2} \tilde{\mathrm{p}}_{p_{0}}^{2}(t, r) d r$

which is included a specific term for estimating the density function of PI hysteresis where $\tilde{p}_{p_{0}}:=\hat{p}_{p_{0}}(t, r)-p_{p_{\max }}$, with $\hat{p}_{p_{0}}(t, r)$ being the estimation of $p_{p_{0}}(r) ; p_{p_{\max }}:=\left(p_{\max } / p_{0}\right)$, and $\gamma_{p r}$ is positive design parameters [12]. As like Eq. (47)Eq. (50) based on Ito formula results:

$$
\begin{aligned}
L V_{n} \leq & \overline{L V_{(n-1)}}+\frac{1}{4} g_{(n-1)} S_{n}^{4} \\
& +S_{n}^{3}\left(g_{n} p_{0} v(t)+f_{n}+\frac{1}{\epsilon_{n}}\left(z_{n}-\bar{x}_{n}\right)\right) \\
& +\underbrace{\frac{3}{2} S_{n}^{2} \psi_{n}^{T} \psi_{n}}_{*}+y_{n}^{3}\left(-\frac{y_{n}}{\epsilon_{n}}+B_{n}\right) \\
& +\frac{3}{2} y_{n} T r\left\{G_{n}^{T} G_{n}\right\}-g_{n} p_{0}\left|S_{n}^{3}\right| \int_{0}^{R} p_{p_{0}}(t, r)\left|F_{r}[v](t)\right| d r \\
& +\frac{b_{M}}{\gamma_{p r}} \int_{0}^{R} \tilde{p}_{p_{0}}(t, r) \frac{\partial}{\partial t} \hat{p}(t, r) d r
\end{aligned}
$$

Using Eq. (58) for the term '*' in Eq. (94), we have:

$\frac{3}{2} S_{n}^{2} \psi_{n}^{T} \psi_{n} \leq \frac{3}{2} n S_{n}^{4} \varphi_{n n}^{2}+\frac{3}{4} r_{n}^{2}+\frac{3}{4} r_{n}^{-2} n^{2} S_{n}^{4}\left(\sum_{j=1}^{n-1} S_{j}^{2} \varphi_{i j}^{2}\right)^{2}$

Combining the above derivations with the auxiliary function $\overline{\mathrm{f}}_{n}$ defined as follows gives:

$$
\begin{aligned}
\overline{\mathrm{f}}_{n}=f_{n} & +\frac{1}{\epsilon_{n}}\left(z_{n}-\frac{-}{\mathrm{x}_{n}}\right)+\frac{3}{2} n S_{n} \varphi_{n n}^{2} \\
+ & \frac{3}{4} r_{n}^{-2} n^{2} S_{n}\left(\sum_{j=1}^{n-1} S_{j}^{2} \varphi_{i j}^{2}\right)^{2}+\frac{1}{4} g_{(n-1)} S_{n}+\frac{3}{4} g_{n}^{\frac{4}{3}} S_{n} \\
\mathrm{LV}_{n} \leq & \overline{\mathrm{LV}_{(n-1)}}+S_{n}^{3}(\underbrace{g_{n} p_{0}}_{g_{n}} v(t)+\overline{f_{n}})-\frac{3}{4} \overline{g_{n}} S_{n}+\frac{3}{4} r_{n}^{2} \\
& +y_{n}^{3}\left(-\frac{y_{n}}{\epsilon_{n}}+B_{n}\right)+\frac{3}{2} y_{n} \operatorname{Tr}\left\{G_{n}^{T} G_{n}\right\} \leq \overline{L V_{(n-1)}} \\
& +\bar{g}_{n} S_{n}^{3}(v(t)+\underbrace{\overline{g_{n}}-1 \bar{f}_{n}}_{\text {unknownterm }}) \\
& -\frac{3}{4} \overline{g_{n}^{3}} S_{n}+\frac{3}{4} r_{n}^{2}+y_{n}^{3}\left(-\frac{y_{n}}{\epsilon_{n}}+B_{n}\right) \\
& +\frac{3}{2} y_{n} \operatorname{Tr}\left\{G_{n}^{T} G_{n}\right\}-\underbrace{g_{n} p_{0}}_{g_{n}}\left|S^{3}\right| \int_{0}^{R} p_{p_{0}}(t, r)\left|F_{r}[v](t)\right| d r \\
& +\frac{b_{M}}{\gamma_{p r}} \int_{0}^{R} \tilde{p}_{p_{0}}(t, r) \frac{\partial}{\partial t} \hat{p}(t, r) d r
\end{aligned}
$$

where $\overline{\mathrm{g}}_{n}=g_{n} p_{0}$. As in Eq. (61), the unknown term in Eq. (96) is approximated using Eq. (53). A simple substitution in Eq. (96) gives: 


$$
\begin{aligned}
\mathrm{LV}_{n} \leq & \overline{\mathrm{LV}_{(n-1)}}+\overline{g_{n}} S_{n}^{3}\left(v(t)+h_{n}\left(Z_{n}\right)\right)-\frac{3}{4} \overline{g_{n}{ }^{3}} S_{n}+\frac{3}{4} r_{n}^{2} \\
& +y_{n}^{3}\left(-\frac{y_{n}}{\epsilon_{n}}+B_{n}\right)+\frac{3}{2} y_{n} \operatorname{Tr}\left\{G_{n}^{T} G_{n}\right\} \leq \overline{L V_{(n-1)}}+b_{m} S_{n}^{3} v(t) \\
& +\underbrace{\overline{g_{n}} S_{n}^{3} h_{n}\left(Z_{n}\right)}_{*}-\frac{3}{4} \overline{g_{n}{ }^{3}} S_{n}+\frac{3}{4} r_{n}^{2}+y_{n}^{3}\left(-\frac{y_{n}}{\epsilon_{n}}+B_{n}\right) \\
& +\frac{3}{2} y_{n} T r\left\{G_{n}^{T} G_{n}\right\}-\overline{g_{n}}\left|S_{n}^{3}\right| \int_{0}^{R} p_{p_{0}}(t, r)\left|F_{r}[v](t)\right| d r \\
& +\frac{b_{M}}{\gamma_{p r}} \int_{0}^{R} \tilde{p}_{p_{0}}(t, r) \frac{\partial}{\partial t} \hat{p}(t, r) d r
\end{aligned}
$$

Applying the same technique used in Eq. (62) to the '*' term in Eq. (104) leads to:

$$
\begin{aligned}
& L V_{n} \leq \underset{\operatorname{LV} V_{(n-1)}}{-}+b_{m} S_{n}^{3} v(t)+\frac{b_{m}}{2 a_{n}^{2}} S_{n}^{6} \theta \zeta_{n}^{T}\left(Z_{n}\right) \zeta_{n}\left(Z_{n}\right)+\frac{1}{4} \varepsilon_{n}^{4} \\
& -\frac{-}{\mathrm{g}_{n}}\left|S_{n}^{3}\right| \int_{0}^{R} p_{p_{0}}(t, r)\left|F_{r}[v](t)\right| d r+\frac{3}{2} y_{n} \operatorname{Tr}\left\{G_{n}^{T} G_{n}\right\} \\
& \quad+\frac{1}{2} a_{n}^{2} b_{M}^{2}+\frac{b_{M}}{\gamma_{p r}} \int_{0}^{R} \widetilde{\mathrm{p}}_{p_{0}}(t, r) \frac{\partial}{\partial t} \widehat{p}(t, r) d r+\frac{3}{4} r_{n}^{2} \\
& \quad+y_{n}^{3}\left(-\frac{y_{n}}{\epsilon_{n}}+B_{n}\right)
\end{aligned}
$$

Choosing $v(t)$ as follows:

$$
\begin{aligned}
v(t)= & -k_{n} S_{n}-\frac{1}{2 a_{n}^{2}} S_{n}^{3} \widehat{\theta} \zeta_{n}^{T}\left(Z_{n}\right) \zeta\left(Z_{n}\right) \\
& +\int_{0}^{R} \widehat{p}_{p_{0}}(t, r)\left|F_{r}[v](t)\right| d r
\end{aligned}
$$

Integrating the above equations using Eq. (71) gives:

$$
\begin{aligned}
L V_{n} & \leq\left(-\sum_{j=1}^{(n-1)} c_{j} S_{j}^{4}+\frac{b_{m}}{\lambda} \tilde{\theta}\left(\sum_{j=1}^{(n-1)} \frac{\lambda}{2 a_{j}^{2}} S_{j}^{6} \zeta_{j}^{T}\left(Z_{j}\right) \zeta_{j}\left(Z_{j}\right)-\dot{\hat{\theta}}\right)\right. \\
& +\frac{1}{2} \sum_{j=1}^{(n-1)} a_{j}^{2} b_{M}^{2}+\frac{1}{4} \sum_{j=1}^{(n-1)} \varepsilon_{j}^{4} \\
& +\sum_{j=2}^{(n-1)}\left(\frac{b_{M}}{4}+\frac{3}{4}\left(\xi_{j} M_{j}\right)^{\frac{4}{3}}+\frac{3}{4}\left(\vartheta_{j} N_{j}\right)^{2}-\frac{1}{\epsilon_{j}}\right) y_{j}^{4} \\
& \left.+\sum_{j=2}^{(n-1)}\left(\frac{1}{4 \xi_{j}^{4}}+\frac{3}{4 \vartheta_{j}^{2}}\right)+\frac{3}{4} \sum_{j=2}^{(n-1)} r_{j}^{2}+\frac{1}{4} b_{M} y_{(i+1)}^{4}\right) \\
& -c_{n} S_{n}^{4}+\frac{b_{m}}{2 a_{n}^{2}} S_{n}^{6} \sim \zeta_{n}^{T}\left(Z_{n}\right) \zeta_{n}\left(Z_{n}\right)+\frac{1}{2} a_{n}^{2} b_{M}^{2}+\frac{1}{4} \varepsilon_{n}^{4} \\
& +\frac{3}{4} r_{n}^{2}+y_{n}^{3}\left(-\frac{y_{n}}{\epsilon_{n}}+B_{n}\right)+\frac{3}{2} y_{n} T r\left\{G_{n}^{T} G_{n}\right\} \\
& +\frac{b_{M}}{\gamma_{p r}} \int_{0}^{R} \tilde{\mathrm{p}}_{p_{0}}(t, r)\left(\frac{\partial}{\partial t} \widehat{p}(t, r)+\gamma_{p r}\left|S_{n}^{3}\right|\left|F_{r}[v](t)\right|\right) d r
\end{aligned}
$$

By choosing $\frac{\partial}{\partial t} \widehat{p}(t, r)$ as follows, we obtain [12]:

$$
\begin{aligned}
& \frac{\partial}{\partial t} \hat{p}_{p_{0}}(t, r)= \begin{cases}-\gamma_{p r}\left[\left|S_{n}^{3}\right|\left|F_{r}[u](t)\right|+\sigma \hat{p}_{p_{0}}(t, r)\right], & 0 \leq \hat{p}_{p_{0}} \leq p_{\max } \\
-\sigma \hat{p}_{p_{0}}(t, r), & \hat{p}_{p_{0}}>p_{\max }\end{cases} \\
& L V_{n} \leq\left(-\sum_{j=1}^{(n-1)} c_{j} S_{j}^{4}+\frac{b_{m}}{\lambda} \tilde{\theta}\left(\sum_{j=1}^{(n-1)} \frac{\lambda}{2 a_{j}^{2}} S_{j}^{6} \zeta_{j}^{T}\left(Z_{j}\right) \zeta_{j}\left(Z_{j}\right)-\dot{\hat{\theta}}\right)\right. \\
& \quad+\frac{1}{2} \sum_{j=1}^{(n-1)} a_{j}^{2} b_{M}^{2}+\frac{1}{4} \sum_{j=1}^{(n-1)} \varepsilon_{j}^{4} \\
& \quad+\sum_{j=2}^{(n-1)}\left(\frac{b_{M}}{4}+\frac{3}{4}\left(\xi_{j} M_{j}\right)^{\frac{4}{3}}+\frac{3}{4}\left(\vartheta_{j} N_{j}\right)^{2}-\frac{1}{\epsilon_{j}}\right) y_{j}^{4} \\
& \left.\quad+\sum_{j=2}^{(n-1)}\left(\frac{1}{4 \xi_{j}^{4}}+\frac{3}{4 \vartheta_{j}^{2}}\right)+\frac{3}{4} \sum_{j=2}^{(n-1)} r_{j}^{2}+\frac{1}{4} b_{M} y_{(i+1)}^{4}\right)-c_{n} S_{n}^{4} \\
& \quad+\frac{b_{m}}{2 a_{n}^{2}} S_{n}^{6} \sim \zeta_{n}^{T}\left(Z_{n}\right) \zeta_{n}\left(Z_{n}\right)+\frac{1}{2} a_{n}^{2} b_{M}^{2}+\frac{1}{4} \varepsilon_{n}^{4}+\frac{3}{4} r_{n}^{2} \\
& \quad+y_{n}^{3}\left(-\frac{y_{n}}{\epsilon_{n}}+B_{n}\right)+\frac{3}{2} y_{n} \operatorname{Tr}\left\{G_{n}^{T} G_{n}\right\}
\end{aligned}
$$

where $\sigma$ is a positive design parameter. As it is shown, Eq. (65) is an adaptive way to directly estimate the density function of PI hysteresis. Using this technique makes the expression in Eq. (107) almost identical to the expression in Eq. (67). Hence, the remaining steps of the stability analysis procedure are similar to the similar expressions of Eq. (67) and result in the same ultimately uniformly boundedness in probability. It is therefore concluded that, according to Lemma 1 of Appendix 1 and using the virtual control input signals (19), the designed control input signal, $v(t)$, in Eq. (106), the $(n-1)$ first-order filters (21), the 
adaptive law of RBFNN weights (22), $\widehat{\theta}(t)$, and the adaptive law of density function Eq. (107) all of the closedloop signals remain bounded in sense of the probability, and the output of the system $y=x_{1}$ tracks the reference input signal of the system, $y_{r}$, with an arbitrarily small error. Thus, the proposed controller design method guarantees the closed-loop stability of the entire system in the presence on unknown actuator hysteresis and the system dynamics. It should be noted that the key point in this design method is the direct estimation of the PI integral via estimating of its density function and involving the associated term in the control input signal Eq. (107).

Funding Open access funding provided by Linköping University. Mr. Mohammad Mahdi Aghajary has received research grants from National Iranian Gas Company (NIGC).

\section{Compliance with ethical standards}

Conflict of interest The authors declare that they have no conflict of interest.

Open Access This article is licensed under a Creative Commons Attribution 4.0 International License, which permits use, sharing, adaptation, distribution and reproduction in any medium or format, as long as you give appropriate credit to the original author(s) and the source, provide a link to the Creative Commons licence, and indicate if changes were made. The images or other third party material in this article are included in the article's Creative Commons licence, unless indicated otherwise in a credit line to the material. If material is not included in the article's Creative Commons licence and your intended use is not permitted by statutory regulation or exceeds the permitted use, you will need to obtain permission directly from the copyright holder. To view a copy of this licence, visit http://creativecommons. org/licenses/by/4.0/.

\section{References}

1. Boskovic JD, Jackson JA, Mehra RK, Nguyen NT (2009) Multiple-model adaptive fault-tolerant control of a planetary lander. J Guid Control Dyn 32(6):1812-1826

2. Zhang X, Parisini T, Polycarpou MM (2004) Adaptive fault-tolerant control of nonlinear uncertain systems: an informationbased diagnostic approach. IEEE Trans Autom Control 49(8): 1259-1274

3. Blanke M, Kinnaert M, Lunze J, Staroswiecki M, Schröder J (2006) Diagnosis and fault-tolerant control, vol 2. Springer, Denmark

4. Gao Z, Ding SX, Cecati C (2015) Real-time fault diagnosis and fault-tolerant control. IEEE Trans Industr Electron 62(6):3752-3756

5. Wang W, Wen C (2011) Adaptive compensation for infinite number of actuator failures or faults. Automatica 47(10):2197-2210

6. Cecati C (2015) A survey of fault diagnosis and fault-tolerant techniques-Part II: Fault diagnosis with knowledge-based and hybrid/active approaches. IEEE Trans Ind Electron 62(6):3757-3767
7. Gao Z, Cecati C, Ding SX (2015) A survey of fault diagnosis and fault-tolerant techniques-part I: fault diagnosis with modelbased and signal-based approaches. IEEE Trans Ind Electron 62(6):3757-3767

8. Ma H, Liang H, Zhou Q, Ahn CK (2018) Adaptive dynamic surface control design for uncertain nonlinear strict-feedback systems with unknown control direction and disturbances. IEEE Trans Syst Man Cybern Syst 99:1-10. https://doi.org/10.1109/ TSMC.2018.2855170

9. Wang D, Huang J (2005) Neural network-based adaptive dynamic surface control for a class of uncertain nonlinear systems in strict-feedback form. IEEE Trans Neural Networks 16(1):195-202. https://doi.org/10.1109/TNN.2004.839354

10. Liu H, Pan Y, Cao J (2020) Composite learning adaptive dynamic surface control of fractional-order nonlinear systems. IEEE Trans Cybern 50(6):2557-2567. https://doi.org/10.1109/TCYB.2019. 2938754

11. Ren B, Ge SS, Su C-Y, Lee TH (2009) Adaptive neural control for a class of uncertain nonlinear systems in pure-feedback form with hysteresis input. IEEE Trans Syst Man Cybern Part B 39(2):431-443. https://doi.org/10.1109/tsmcb.2008.2006368

12. Zhang X, Su C-Y, Lin Y, Ma L, Wang J (2015) Adaptive neural network dynamic surface control for a class of time-delay nonlinear systems with hysteresis inputs and dynamic uncertainties. IEEE Trans Neural Netw Learn Syst 26(11):2844-2860. https:// doi.org/10.1109/TNNLS.2015.2397935

13. Su C-Y, Wang Q, Chen X, Rakheja S (2005) Adaptive variable structure control of a class of nonlinear systems with unknown Prandtl-Ishlinskii hysteresis. IEEE Trans Autom Control 50(12):2069-2074. https://doi.org/10.1109/TAC.2005.860260

14. Wang Q, Su C-Y (2006) Robust adaptive control of a class of nonlinear systems including actuator hysteresis with PrandtlIshlinskii presentations. Automatica 42(5):859-867. https://doi. org/10.1016/j.automatica.2006.01.018

15. Zhang X, Lin Y, Mao J (2011) A robust adaptive dynamic surface control for a class of nonlinear systems with unknown PrandtlIshilinskii hysteresis. Int $\mathrm{J}$ Robust Nonlinear Control 21(13):1541-1561. https://doi.org/10.1002/rnc.1652

16. Zhang T-P, Ge SS (2008) Adaptive dynamic surface control of nonlinear systems with unknown dead zone in pure feedback form. Automatica 44(7):1895-1903. https://doi.org/10.1016/j. automatica.2007.11.025

17. Li Z, Li T, Feng G (2016) Adaptive neural control for a class of stochastic nonlinear time-delay systems with unknown dead zone using dynamic surface technique. Int J Robust Nonlinear Control 26(4):759-781. https://doi.org/10.1002/rnc.3336

18. Si W-J, Dong X-D, Yang F-F (2017) Adaptive neural dynamic surface control for a general class of stochastic nonlinear systems with time delays and input dead-zone. Int $\mathrm{J}$ Control Autom Syst 15(5):2416-2424. https://doi.org/10.1007/s12555-016-0564-y

19. Wang HQ, Chen B, Lin C (2013) Adaptive neural tracking control for a class of stochastic nonlinear systems with unknown dead-zone. Int J Innov Comput Inf Control 9(8):3257-3269

20. Wang H, Chen B, Lin C (2013) Direct adaptive neural tracking control for a class of stochastic pure-feedback nonlinear systems with unknown dead-zone. Int $\mathbf{J}$ Adapt Control Signal Process 27(4):302-322

21. Wang H, Chen B, Liu K, Liu X, Lin C (2014) Adaptive neural tracking control for a class of nonstrict-feedback stochastic nonlinear systems with unknown backlash-like hysteresis. IEEE transactions on neural networks learning systems 25(5):947-958. https://doi.org/10.1109/tnnls.2013.2283879

22. Ji G (2012) Adaptive neural network dynamic surface control for perturbed nonlinear time-delay systems. Int $\mathrm{J}$ Autom Comput 9(2):135-141. https://doi.org/10.1007/s11633-012-0626-4 
23. G. Liu et al. (2020) adaptive neural network dynamic surface control algorithm for pneumatic servo system. In: Proceedings of the 11th international conference on modelling, identification and control (ICMIC2019), pp. 821-829, doi: https://doi.org/10.1007/ 978-981-15-0474-7_77

24. D. Gao, Z. Sun, and T. Du, (2007) Dynamic surface control for hypersonic aircraft using fuzzy logic system. In: 2007 IEEE international conference on automation and logistics (pp. 2314-2319)

25. Wang H, Chen B, Lin C (2012) Direct adaptive neural control for strict-feedback stochastic nonlinear systems. Nonlinear Dyn 67(4):2703-2718

26. Rodnishchev N and Somov Y (2018) control optimization in aerospace engineering at stochastic perturbations and stream of faults. In: 2018 5th IEEE International Workshop on Metrology for AeroSpace (MetroAeroSpace), Rome, 2018, pp. 171-175. https://doi.org/10.1109/MetroAeroSpace.2018.8453602

27. N. Rodnishchev and Y. Somov, (2018) Control optimization in aerospace engineering at stochastic perturbations and stream of faults. In: 5th IEEE international workshop on metrology for aerospace, metroaerospace 2018 - proceedings, Aug (pp. 171-175) doi: https://doi.org/10.1109/MetroAeroSpace.2018. 8453602

28. Wu J, Chen X, Zhao Q, Li J, Wu Z-G (2020) Adaptive neural dynamic surface control with prespecified tracking accuracy of uncertain stochastic nonstrict-feedback systems. IEEE Trans Cybern. https://doi.org/10.1109/TCYB.2020.3012607

29. Chen P, Zhang T (2020) Adaptive dynamic surface control of stochastic nonstrict-feedback constrained nonlinear systems with input and state unmodeled dynamics. Int J Adapt Control Signal Process 34(10):1405-1429

30. S. Liu, X. Sun, W. Dong, and H. Song, (2009) Control law design of aircraft super-maneuverable flight based on dynamic surface backstepping control. In: 2009 Chinese control and decision conference, pp. 1764-1768, doi: https://doi.org/10.1109/ccdc. 2009.5192368

31. D. Wang, Z. Peng, G. Sun, and H. Wang, (2012) Adaptive dynamic surface control for coordinated target tracking of autonomous surface vehicles using neural networks. In: proceedings of the 31st Chinese Control Conference (pp. 2871-2876), doi: https://doi.org/https://doi.org/10.1007/s11071014-1277-5

32. N. Wang, Z. Liu, Z. Zheng, and M. J. Er, (2018) Global Exponential Trajectory Tracking Control of Underactuated Surface Vehicles Using Dynamic Surface Control Approach. In: 2018 International Conference on Intelligent Autonomous Systems (ICoIAS), pp. 221-226, doi: https://doi.org/10.1109/ICoIAS. 2018.8494037

33. D. Caguiat, J. Scharschan, D. Zipkin, and J. Nicolo, (2006) Applied neural network for navy marine gas turbine stall algorithm development. In: 2006 IEEE aerospace conference, p. 15 pp

34. W. Li, T. Lan, and W. Lin, (2010) Nonlinear adaptive robust governor control for turbine generator. In: IEEE ICCA 2010, pp. $820-825$

35. X. Tan, X. Su, K. Zhao, and M. Tan, (2016) Robust adaptive backstepping control of micro-turbines. In: 2016 Chinese control and decision conference (CCDC), pp. 490-493

36. Liu X, Su C-Y, Yang F (2017) FNN approximation-based active dynamic surface control for suppressing chatter in micro-milling with piezo-actuators. IEEE Trans Syst Man Cybern Syst 47(8):2100-2113

37. Lei D, Wang T, Cao D, Fei J (2016) Adaptive dynamic surface control of MEMS gyroscope sensor using fuzzy compensator.
IEEE Access 4:4148-4154. https://doi.org/10.1109/ACCESS. 2016.2596538

38. Xu B (2017) Disturbance observer-based dynamic surface control of transport aircraft with continuous heavy cargo airdrop. IEEE Trans Syst Man Cybern Syst 47(1):161-170. https://doi.org/10. 1109/TSMC.2016.2558098

39. Tang X, Tao G, Joshi SM (2003) Adaptive actuator failure compensation for parametric strict feedback systems and an aircraft application. Automatica 39(11):1975-1982

40. Tao G, Joshi SM, Ma X (2001) Adaptive state feedback and tracking control of systems with actuator failures. IEEE Trans Autom Control 46(1):78-95

41. Fan H, Liu B, Shen Y, Wang W (2014) Adaptive failure compensation control for uncertain systems with stochastic actuator failures. IEEE Trans Autom Control 59(3):808-814

42. Corradini ML, Orlando G (2007) Actuator failure identification and compensation through sliding modes. IEEE Trans Control Syst Technol 15(1):184-190

43. Wang W, Wen C (2010) Adaptive actuator failure compensation control of uncertain nonlinear systems with guaranteed transient performance. Automatica 46(12):2082-2091

44. Tang X, Tao G, Joshi SM (2007) Adaptive actuator failure compensation for nonlinear MIMO systems with an aircraft control application. Automatica 43(11):1869-1883

45. Krstic M, Kanellakopoulos I, Kokotovic Pv (1995) Nonlinear and adaptive control design, vol 222. Wiley, New York

46. Zhang L, Yang G-H (2020) Adaptive sensor and actuator failure compensation for $\mathrm{H} \propto$ static output control of linear systems: a new Lyapunov function method. Int J Syst Sci 51(1):146-157

47. Zhu F, Zhang X, Li H (2020) Actuator failure compensation control scheme of the nonlinear triangular systems by static gain technique. Int J Control Autom Syst 18(9):2297-2305

48. Nai Y, Yang Q, Wu Z (2020) Prescribed performance adaptive neural compensation control for intermittent actuator faults by state and output feedback. IEEE Trans Neural Netw Learn Syst. https://doi.org/10.1109/TNNLS.2020.3026208

49. Zhang L, Yang G-H (2020) Adaptive fuzzy fault compensation tracking control for uncertain nonlinear systems with multiple sensor faults. Fuzzy Sets Syst 392:46-59

50. D. Swaroop, J. C. Gerdes, P. P. Yip, and J. K. Hedrick, (1997) Dynamic surface control of nonlinear systems. In: American control conference, 1997. Proceedings of the 1997, vol. 5, pp. 3028-3034, doi: https://doi.org/10.1109/acc.1997.612013

51. Swaroop D, Hedrick JK, Yip PP, Gerdes JC (2000) Dynamic surface control for a class of nonlinear systems. IEEE Trans Autom Control 45(10):1893-1899. https://doi.org/10.1109/tac. 2000.880994

52. Wu GQ, Song SM, Sun JG (2018) Adaptive dynamic surface control for spacecraft terminal safe approach with input saturation based on tracking differentiator. Int J Control Autom Syst 16(3):1129-1141. https://doi.org/10.1007/s12555-017-0531-2

53. Li G, Xu W, Zhao J, Wang S, Li B (2017) Precise robust adaptive dynamic surface control of permanent magnet synchronous motor based on extended state observer. IET Sci Meas Technol 11(5):590-599. https://doi.org/10.1049/iet-smt.2016.0252

54. Mao X, Yuan C (2006) Stochastic differential equations with Markovian switching. Imperial College Press, London

55. Stehlík M, Kisel'ák J, Bukina E, Lu Y, Baran S (2020) Fredholm integral relation between compound estimation and prediction (FIRCEP). Stoch Anal Appl 38(3):427-459

Publisher's Note Springer Nature remains neutral with regard to jurisdictional claims in published maps and institutional affiliations. 